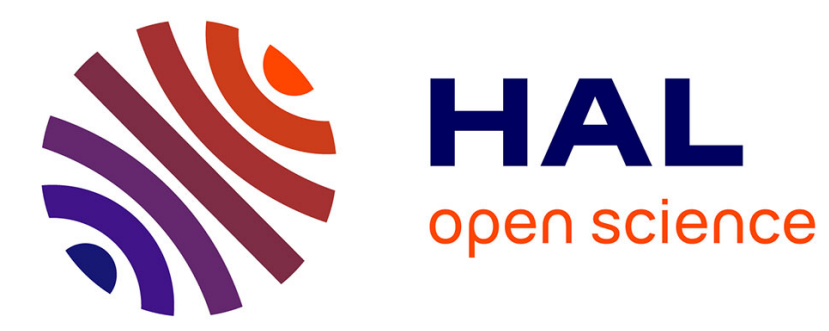

\title{
Proof of quasipatterns for the Swift-Hohenberg equation
} Boele Braaksma, Gérard Iooss, Laurent Stolovitch

\section{To cite this version:}

Boele Braaksma, Gérard Iooss, Laurent Stolovitch. Proof of quasipatterns for the Swift-Hohenberg equation. Communications in Mathematical Physics, 2017, 353 (1), pp.37 - 67. 10.1007/s00220-0172878-x . hal-01891588

\section{HAL Id: hal-01891588 \\ https://hal.science/hal-01891588}

Submitted on 9 Oct 2018

HAL is a multi-disciplinary open access archive for the deposit and dissemination of scientific research documents, whether they are published or not. The documents may come from teaching and research institutions in France or abroad, or from public or private research centers.
L'archive ouverte pluridisciplinaire HAL, est destinée au dépôt et à la diffusion de documents scientifiques de niveau recherche, publiés ou non, émanant des établissements d'enseignement et de recherche français ou étrangers, des laboratoires publics ou privés. 


\title{
Proof of quasipatterns for the Swift-Hohenberg equation
}

\author{
Boele Braaksma*, Gérard Iooss ${ }^{\dagger}$ and Laurent Stolovitch ${ }^{\ddagger}$
}

February 28, 2017

\begin{abstract}
This paper establishes the existence of quasipatterns solutions of the Swift-Hohenberg PDE. In a former approach [BIS], we avoided the use of Nash-Moser scheme, but our proof contains a gap. The present proof of existence is based on the works by Berti et al [BBP10], [BB10], [BCP] related to the Nash-Moser scheme. For solving the small divisor problem, we need to introduce a new free parameter related to the freedom in the choice of parameterization of the bifurcating solution. Thanks to a transversality condition, the result gives only a bifurcating set, located in a small hornlike region centered on a curve, with the origin at the bifurcation point.

Keywords: Bifurcations, quasipatterns, small divisors, Nash-Moser scheme
\end{abstract}

AMS: 35B32, 52C23, 35C20, 37J40, 58C15

\section{Contents}

1 Introduction $\quad 2$

2 Setting of the problem $\quad 5$

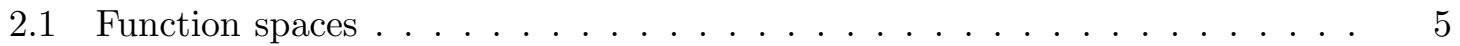

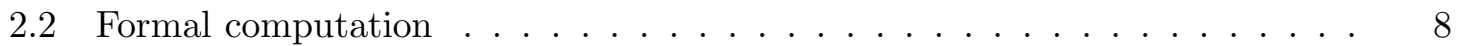

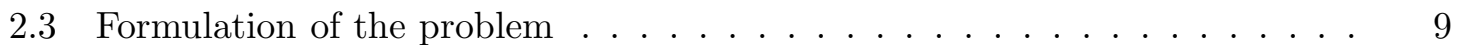

2.4 Strategy . . . . . . . . . . . . . . . . . . . 12

2.5 Tame properties . . . . . . . . . . . . . . . . . 13

2.6 Estimate of $\left(\Pi_{N} \Pi_{0} \mathcal{L}_{\epsilon, \lambda^{\prime}, \nu, V} \Pi_{N} \Pi_{0}\right)^{-1}$ in $\mathcal{H}_{s}$ for small $N \ldots \ldots \ldots 14$

2.7 Estimate of $\left(\Pi_{N} \Pi_{0} \mathcal{L}_{\epsilon, \lambda^{\prime}, \nu, V} \Pi_{N} \Pi_{0}\right)^{-1}$ in $\mathcal{H}_{0}$ for large $N \ldots \ldots \ldots$

3 Estimate of the inverse linearized operator in $\mathcal{H}_{s} \quad 19$

3.1 Separation properties $(\mathrm{H} 1)$ and $(\mathrm{H} 2) \ldots \ldots \ldots \ldots$

3.2 Estimate in $\Pi_{N} \Pi_{0} \mathcal{H}_{s}$ of $\left(\Pi_{0} \Pi_{N} \mathcal{L}_{\epsilon, \lambda^{\prime}, \nu, V} \Pi_{N} \Pi_{0}\right)^{-1} \ldots \ldots \ldots 22$

${ }^{*}$ University of Groningen, Johann Bernoulli Institute, P.O.Box 407, 9700 AK Groningen, The Netherlands. E-mail : B.L.J.Braaksma@rug.nl

${ }^{\dagger}$ Université Côte d'Azur, CNRS, LJAD, France. E-mail : gerard.iooss@unice.fr

${ }^{\ddagger}$ CNRS, Université Côte d'Azur, LJAD, France. E-mail : stolo@unice.fr. Research of G.Iooss and L.Stolovitch was supported by ANR grant "ANR-10-BLAN 0102" for the project DynPDE. Research of L.Stolovitch was supported by ANR grant "ANR-14-CE34-0002-01" for the project "Dynamics and CR geometry". 


\section{Introduction}

The object of the paper is the existence of a special kind of stationary solutions (i.e. independent of $t$ ), bifurcating from 0 (i.e. tending towards zero when the parameter $\lambda$ tends towards 0), called quasipatterns of the 2-dimensional Swift-Hohenberg PDE

$$
\frac{\partial U}{\partial t}=\lambda U-(1+\Delta)^{2} U-U^{3}
$$

where $U$ is the unknown real-valued function on $\mathbb{R}^{+} \times \mathbb{R}^{2}, \Delta:=\left(\frac{\partial^{2}}{\partial x_{1}^{2}}+\frac{\partial^{2}}{\partial x_{2}^{2}}\right)$ and $\lambda$ is a parameter. We are interested in two-dimensional patterns that have no translation symmetry and are quasiperiodic in any spatial direction.

Mathematical existence of quasipatterns is one of the outstanding problems in pattern formation theory. To our knowledge, hereafter is the first proof of existence of such quasipatterns of a PDE. Quasipatterns were discovered in nonlinear pattern-forming systems in the Faraday wave experiment [BCM92, EF94], in which a layer of fluid is subjected to vertical oscillations. Since their discovery, they have also been found in nonlinear optical systems, shaken convection and in liquid crystals (see references in [AG12]). In spite of the lack of translation symmetry (in contrast to periodic patterns), the solutions are $\pi / q$-rotation invariant for some integer $q$ (most often observed, $2 q$ is 8,10 or 12 ).

In many of these experiments, the domain is large compared with the size of the pattern, and the boundaries appear to have little effect. Furthermore, the pattern is usually formed in two directions $\left(x_{1}\right.$ and $\left.x_{2}\right)$, while the third direction $(z)$ plays little role. Mathematical models of the experiments are therefore often posed with two unbounded directions, and the basic symmetry of the problem is $E(2)$, the Euclidean group of rotations, translations and reflections of the $\left(x_{1}, x_{2}\right)$ plane.

The above model equation is the simplest pattern-forming PDE, and is extremely successful for describing primary bifurcations (the first symmetry breaking) of hydrodynamical instability problems such as the Rayleigh - Bénard convection. Its essential properties are that

i) the system is invariant under the group $E(2)$;

ii) the instability occurs for a certain critical value of the parameter (here $\lambda=0$ ) for which critical modes are given by wave vectors sitting on a circle of non zero radius (here the unit circle);

iii) the linear part is selfadjoint and contains the main derivatives.

The steady Swift-Hohenberg equation reads

$$
(1+\Delta)^{2} U-\lambda U+U^{3}=0 .
$$

The parameter $\lambda$ is supposed to be real and small in absolute value. The solutions we are interested in should tend towards zero as the parameter goes to zero. 


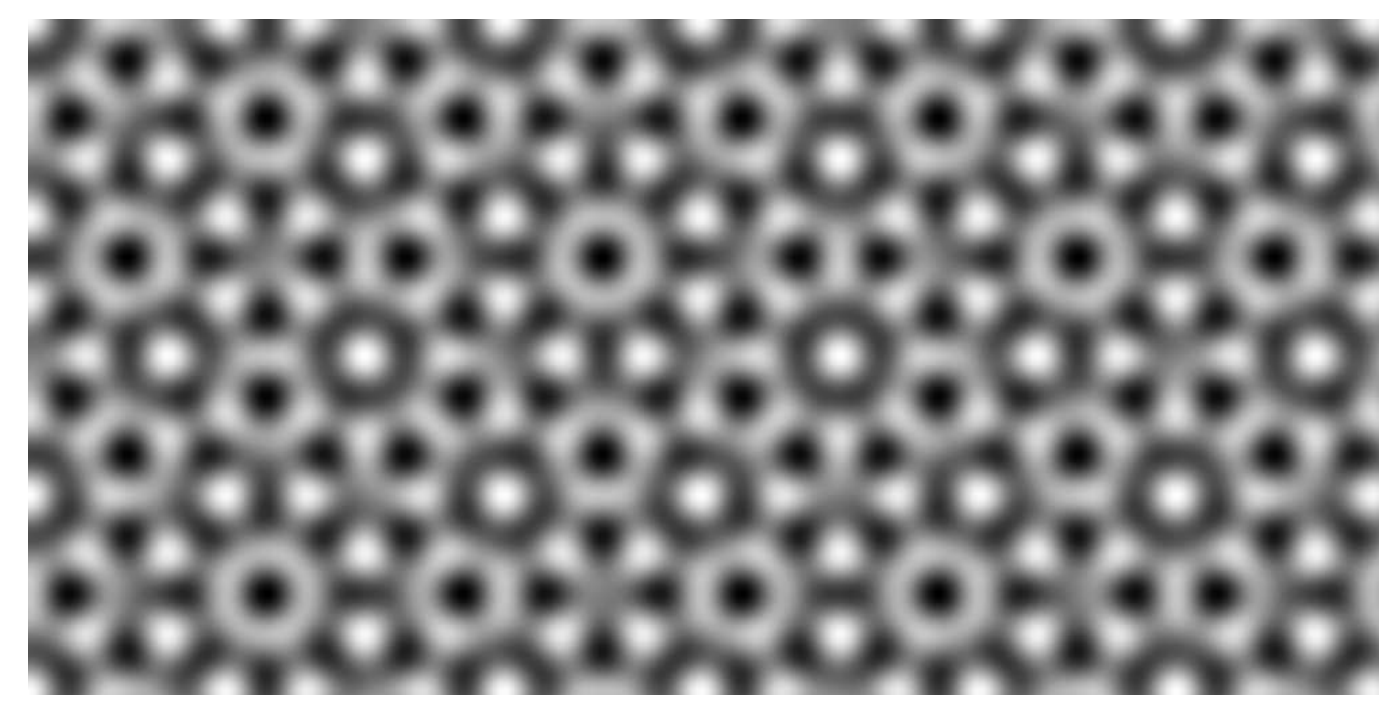

Figure 1: Example 8-fold quasipattern after [IR10]. This is an approximate solution of the steady Swift-Hohenberg equation (2) with $\lambda=0.1$, computed by using Newton iteration to find an equilibrium solution truncated to wavenumbers satisfying $|\mathbf{k}| \leq \sqrt{5}$ and to the quasilattice $\Gamma_{27}$ obtained with $N_{\mathbf{k}} \leq 27$.

We study equation (2) for $\lambda>0$. Namely, let $2 q$ be an even integer and let $\mathbf{k}_{j}=$ $\exp \frac{i \pi(j-1)}{q}, j=1, \ldots, 2 q$ be the $2 q$ unit vectors of the plane, identified with roots of unity. Let $\Gamma$ be the lattice of linear combinations of vectors $\mathbf{k}_{j}$ with nonnegative integer coefficients. We look for the existence of a (nonzero) $\pi / q$-rotation invariant solution of the form

$$
U(\mathbf{x})=\sum_{\mathbf{k} \in \Gamma} u^{(\mathbf{k})} e^{i \mathbf{k} \cdot \mathbf{x}}
$$

which belongs to a Sobolev space $\mathcal{H}_{s}, s \geq 0$ :

$$
\|U\|_{s}^{2}:=\sum_{\mathbf{k} \in \boldsymbol{\Gamma}}\left|u^{(\mathbf{k})}\right|^{2}\left(1+N_{\mathbf{k}}^{2}\right)^{s}<+\infty .
$$

The natural number $N_{\mathbf{k}}$ denotes a norm of $\mathbf{k}$ in the lattice $\Gamma$, which we define below. We indicate on Figure 1 a computation made on this model equation for $q=4$, keeping only Fourier modes such that $N_{\mathrm{k}} \leq 27$. We then would like to show that such a solution exists indeed, for small positive parameters $\lambda$. Our main result is:

Theorem 1. Let $q \geq 4$ be an integer and let $d$ be the dimension of the $\mathbb{Q}$-vector space spanned by the wave vectors $\mathbf{k}_{j}, j=1, \ldots, 2 q$. Moreover, assume that Condition 2 is verified. Then, there exists $s_{0}>d / 2, \epsilon_{0}>0$, such that, for any $s \geq s_{0}$, and for any $\epsilon$ with $0<\epsilon<\epsilon_{0}$ there exist $\bar{\lambda}_{\epsilon}$ such that the steady Swift-Hohenberg equation, for $\lambda=\lambda_{2} \epsilon^{2}-\epsilon^{4} \bar{\lambda}_{\epsilon}$ admits a quasipattern solution $U$ in $\mathcal{H}_{s}$, invariant under rotations of angle $\pi / q$ of the form

$$
U=\epsilon u_{0}+\epsilon^{3} v(\epsilon)
$$


where $u_{0}$ is given by (16) and where $\lambda_{2}=3(2 q-1)>0, \bar{\lambda}_{\epsilon}$ tends towards $-\lambda_{4}$ as $\epsilon$ tends towards 0 . The quasiperiodic function $u_{0}$ spans the kernel of $(1+\Delta)^{2}$, and coefficients $\lambda_{2}, \lambda_{4}$ occurring in formulae above, are the ones defined in the truncated asymptotic expansion of the solution, computed in section 2.2, see also [IR10]. The set $\bar{\Lambda}_{\epsilon}$ of all such $\bar{\lambda}_{\epsilon}$ is close to $-\lambda_{4}$ and has asymptotic full measure as $\epsilon$ tends to 0.

Condition 2. For $n \in \mathbb{N}$, let $N_{n}:=N_{0}^{2^{n}}$ for some constant $N_{0}=M_{\epsilon_{3}}$ as defined in (42). Let $\mathcal{N}_{n}$ be the dimension of the finite-dimensional subspace $E_{N_{n}}$ of elements (3) such that $N_{\mathbf{k}} \leq N_{n}$ (see definition 9).

We assume that there is a constant $c_{1}>0$ such that, for any $j \in\left\{1,2, \ldots, \mathcal{N}_{n}\right\}$, the following inequality holds for any $0<\epsilon<\epsilon_{3},|\nu| \leq \epsilon_{0}\left|\lambda^{\prime}\right|,\left|\lambda^{\prime}\right| \leq \epsilon_{0}\left|\lambda_{4}\right|$,

$$
\left|\left\langle u_{0}^{2} \zeta_{j}^{\left(N_{n}\right)}\left(\epsilon, \lambda^{\prime}, \nu\right), \zeta_{j}^{\left(N_{n}\right)}\left(\epsilon, \lambda^{\prime}, \nu\right)\right\rangle_{0}-(2 q-1)\right|>c_{1} .
$$

Here, the $\zeta_{j}^{\left(N_{n}\right)}\left(\epsilon, \lambda^{\prime}, \nu\right)^{\prime}$ s are the eigenvectors, with norm 1 , of the linear operator

$$
\Pi_{N_{n}} \Pi_{0} \mathcal{L}_{\epsilon, \lambda^{\prime}, \nu, V\left(\epsilon, \lambda^{\prime}, \nu\right)} \Pi_{N_{n}} \Pi_{0},
$$

where $\mathcal{L}_{\epsilon, \lambda^{\prime}, \nu, V\left(\epsilon, \lambda^{\prime}, \nu\right)}$ is defined in (36), $V \in \mathcal{U}_{M}^{\left(N_{n}\right)}$ (see (44)), and $\Pi_{N}$ is the orthogonal projection on $E_{N}$ and $\Pi_{0}$ the orthogonal projection on the complement of the kernel $\mathbb{R} u_{0}$ of $(1+\Delta)^{2}$.

Remark 3. Condition 2 is a transversality condition. It concerns only eigenvalues of $\mathcal{L}_{\epsilon, \lambda^{\prime}, \nu, V\left(\epsilon, \lambda^{\prime}, \nu\right)}$ which are very close to 0 . In case when these eigenvalues are simple, then Condition 2 is satisfied near $\epsilon=0$ for any $q$ (see Remark 28).

Remark 4. The good set $\bar{\Lambda}_{\epsilon}$ is of asymptotic full measure. This means the following:

$$
\frac{1}{2 \epsilon} \text { meas }\left\{\bar{\Lambda}_{\epsilon} \cap\left(-\lambda_{4}-\epsilon,-\lambda_{4}+\epsilon\right)\right\} \underset{\epsilon \rightarrow 0}{\rightarrow} 1 \text {. }
$$

Remark 5. For $\lambda<0$ the solution $U=0$ is isolated in an open ball of radius $\sqrt{|\lambda|}$ (see [BIS][Remark 3]).

Remark 6. The expression that we obtain for the bifurcating set solution of (2), does not say anything on its structure as a curve $U$ function of $\sqrt{\lambda}$, as it would be the case in usual bifurcation problems. However we show that the bifurcating set $(\lambda, U)$ lies in a hornlike region centered on a curve, with the tip at the bifurcation point (see Remark 29).

One of the main difficulties is that the linearized operator at $U=0$, has an unbounded inverse. Indeed, it is easy to show that the eigenvalues of $(1+\Delta)^{2}$ in $\mathcal{H}_{s}$ are $\left(1-|\mathbf{k}|^{2}\right)^{2}$ where $\mathbf{k} \in \Gamma$. These numbers accumulate at any point of $\mathbb{R}^{+}$. It creates a small divisor problem, such that if $\lambda \geq 0$ nothing can be said a priori about the inverse of $(1+\Delta)^{2}-\lambda I$.

We use the first terms of the asymptotic expansion of the solution and change the unknown as $U=U_{\epsilon}+\epsilon^{2} W$ and $\lambda=\epsilon^{2} \lambda_{2}-\epsilon^{3} \lambda^{\prime}$ for some well chosen $\left(U_{\epsilon}, \lambda_{2}\right), \lambda_{2}$ being positive and for $\left(\epsilon, \lambda^{\prime}\right) \in\left(0, \epsilon_{0}\right) \times[-1,1]$. The parameter $\lambda^{\prime}$ is used here for providing some elasticity to the parameterization of the bifurcating solution, which would be classically parameterized by $\epsilon$ only, in usual bifurcation problems. The choice of the factor $\epsilon^{3}$ in front 
of $\lambda^{\prime}$ means that any $\lambda^{\prime} \in[-1,1]$ does not modify much the relationship between $\lambda$ and $\epsilon$, i.e. the principal part of the bifurcating solution is nearly independent of $\lambda^{\prime}$.

Let $L_{\epsilon, \lambda^{\prime}, W}$ be the linear part at $W$, close to 0 , of the nonlinear equation so obtained. For $\epsilon=0$, the operator $L_{0}=(1+\Delta)^{2}$ is a positive selfadjoint operator in $\mathcal{H}_{s}$. It is bounded from $\mathcal{H}_{s+4}$ into $\mathcal{H}_{s}$, and it is not Fredholm, since its range is not closed. Its spectrum is an essential spectrum filling the half line $[0, \infty)$. The set of eigenvalues is dense in the spectrum. The linear operator $L_{\epsilon, \lambda^{\prime}, W}$ is the sum of $L_{0}$ and a bounded operator (multiplication by a small function $\left.O\left(\epsilon^{2}\right)\right)$ selfadjoint in $\mathcal{H}_{0}$.

Writing $W$ as $\nu u_{0}+3 \nu \epsilon^{2} u_{1}+\epsilon^{2} V$, where $u_{1}$ is a known function and $V$ are orthogonal to the kernel $\mathbb{R} u_{0}$ of $L_{0}$, the projection of (2) onto the range of $L_{0}$ (i.e the orthognal to its kernel) reads $\mathcal{F}\left(\epsilon, \lambda^{\prime}, \nu, V\right)=0$. It is called the range equation. The most difficult part of the problem consists in finding good parameters $\left(\epsilon, \lambda^{\prime}, \nu\right)$ for which there is a solution of the range equation in some Sobolev space. It is solved using a version of the Nash-Moser theorem due to Berti, Bolle and Procesi [BBP10]. To apply such a result, we investigate for $\epsilon>0,\left|\nu / \lambda^{\prime}\right|$ small enough, and $\lambda^{\prime} \in[-1,1]$, not only the invertibility in some $\mathcal{H}_{s}$ but also the bound of the inverses of the "truncated-restricted" of the linear operator $\mathcal{L}_{\epsilon, \lambda^{\prime}, \nu, V}$ which is the differential with respect to $V$ of the projection $\mathcal{F}\left(\epsilon, \lambda^{\prime}, \nu, V\right)$ of $(2)$ on the orthogonal complement of the kernel. The "truncated-restricted" of a linear operator $A$ is the projection onto "small frequencies" of the restriction of $A$ to "small frequencies" (here "small" is in the sense of the norm $N_{\mathbf{k}}, \mathrm{k} \in \Gamma$ ).

For this analysis we use intensively the Nash-Moser technique developed in [BB10], [BBP10], [BCP], which is based here on separation properties of the set $\left\{\left(1-|\mathbf{k}|^{2}\right)^{2} ; \mathbf{k} \in \Gamma\right\}$, the set of eigenvalues of $L_{0}$. Notice that our former approach to the problem [BIS], that avoided the use of Nash-Moser scheme, contains a gap.

Using the main result of [BBP10], we obtain the existence of a $C^{1}$ function $V$ of all parameters and a 3-dimensional set $G$ of parameters over which $V$ is solution of the range equation. The key point is that this set of good parameters $G$ is such that for any $\epsilon \in\left[0, \epsilon_{1}\right]$ we need to choose $\left(\nu, \lambda^{\prime}\right) \in G_{\epsilon}$, where the set $G_{\epsilon}$ is the complement of "bad strips" in the $\left(\nu, \lambda^{\prime}\right)$ plane, and has asymptotically full measure as $\epsilon$ tends towards 0 (see Remark above). It remains to solve the bifurcation equation (i.e. the projection onto the kernel of (2)). Plugging the solution $V$ obtained by the Nash-Moser theorem, we obtain a $C^{1}$ equation linking $\epsilon, \nu$ and $\lambda^{\prime}$. The knowledge of the main part of this equation allows us to solve $\lambda^{\prime}$ as a $C^{1}$ function of $\epsilon$ and $\nu$. For each $\epsilon$, the graph of $\nu \mapsto \lambda^{\prime}(\epsilon, \nu)$ intersects transversally $G_{\epsilon}$, leading to a solution that solves the range equation as well as the bifurcation equation, that is the full nonlinear problem. This solution is $W(\epsilon, \nu):=\nu u_{0}+3 \nu \epsilon^{2} u_{1}+\epsilon^{2} V\left(\epsilon, \lambda^{\prime}(\epsilon, \nu), \nu\right)$.

\section{Setting of the problem}

\section{$2.1 \quad$ Function spaces}

In this section, we introduce the function spaces we use.

Let $q \geq 4$ be an integer. Let us define the unit wave vectors (identifying $\mathbb{C}$ with $\mathbb{R}^{2}$ )

$$
\mathbf{k}_{j}:=e^{i \pi \frac{j-1}{q}}, \quad j=1, \ldots, 2 q .
$$


We define the quasilattice $\Gamma \subset \mathbb{R}^{2}$ to be the set of points spanned by (nonnegative) integer linear combinations of the $k_{j}$ 's :

$$
\mathbf{k}_{m}=\sum_{j=1}^{2 q} m_{j} \mathbf{k}_{j}, \quad \mathbf{m}=\left(m_{1}, \ldots, m_{2 q}\right) \in \mathbb{N}^{2 q} .
$$

We know (see [Wash97]) that the $\mathbb{Q}$ - vector space spanned by $\left\{\mathbf{k}_{j}, j=1,2, . ., 2 q\right\}$ has dimension $d=\varphi(2 q)=2\left(l_{0}+1\right)$ where $\varphi$ is the Euler totient function, and $l_{0}+1$ is the order of the algebraic integer $\omega:=2 \cos \pi / q\left(l_{0}=1\right.$ for $q=4,5,6, l_{0}=2$ for $\left.q=7 \ldots\right)$ with $2\left(l_{0}+1\right) \leq q$. Let us define the subset of the $d$ vectors $\left\{\mathbf{k}_{j}^{*}, j=1,2, . ., d\right\}$ of $\left\{\mathbf{k}_{j}\right.$, $j=1,2, . ., 2 q\}$ which forms a basis. Then

$$
\mathbf{k}_{j}=\sum_{s=1}^{d} \alpha_{j s} \mathbf{k}_{s}^{*}, \alpha_{j s} \in \mathbb{Q} .
$$

and any $\mathbf{k} \in \Gamma$ may be written in two different ways

$$
\mathbf{k}=\sum_{j=1}^{2 q} m_{j} \mathbf{k}_{j}=\sum_{s=1}^{d} r_{s} \mathbf{k}_{s}^{*}, m_{j} \in \mathbb{N}, r_{s} \in \mathbb{Q}
$$

where $r_{s}=\sum_{j=1}^{2 q} m_{j} \alpha_{j s}$.

Let us define $\alpha_{j s}:=\frac{n_{j s}}{d_{j s}}$ with irreducible fractions and

$$
\mathfrak{d}=\text { l.c. } m_{\substack{j=1, . .2 q \\ s=1, . . d}}\left\{d_{j s}\right\}, \text { then } \mathfrak{d} \alpha_{j s}=\beta_{j s} \in \mathbb{Z} .
$$

Remark 7. Notice that we have $\mathfrak{d}=1$ for example for $q=4,5,6,7,8,9,10,11,12$. We can choose $\mathbf{k}_{s}^{*}=\mathbf{k}_{s}, s=1, . ., d$ as this results from

$$
\mathbf{k}_{j+q}=-\mathbf{k}_{j}, j=1, \ldots, q,
$$

for $q=4$ or $8(d=q)$, and from the identities

$$
\begin{aligned}
\mathbf{k}_{5} & =\mathbf{k}_{4}-\mathbf{k}_{3}+\mathbf{k}_{2}-\mathbf{k}_{1} \text { for } q=5 \quad(d=4) \\
\mathbf{k}_{5} & =\mathbf{k}_{3}-\mathbf{k}_{1}, \mathbf{k}_{6}=\mathbf{k}_{4}-\mathbf{k}_{2} \text { for } q=6, \quad(d=4) \\
\mathbf{k}_{7} & =\mathbf{k}_{6}-\mathbf{k}_{5}+\mathbf{k}_{4}-\mathbf{k}_{3}+\mathbf{k}_{2}-\mathbf{k}_{1} \text { for } q=7 \quad(d=6) \\
\mathbf{k}_{7} & =\mathbf{k}_{4}-\mathbf{k}_{1}, \mathbf{k}_{8}=\mathbf{k}_{5}-\mathbf{k}_{2}, \mathbf{k}_{9}=\mathbf{k}_{6}-\mathbf{k}_{3} \text { for } q=9, \quad(d=6) \\
\mathbf{k}_{9} & =\mathbf{k}_{7}-\mathbf{k}_{5}+\mathbf{k}_{3}-\mathbf{k}_{1}, \mathbf{k}_{10}=\mathbf{k}_{8}-\mathbf{k}_{6}+\mathbf{k}_{4}-\mathbf{k}_{2} \text { for } q=10, \quad(d=8) \\
\mathbf{k}_{11} & =\mathbf{k}_{10}-\mathbf{k}_{9}+\mathbf{k}_{8}-\mathbf{k}_{7}+\mathbf{k}_{6}-\mathbf{k}_{5}+\mathbf{k}_{4}-\mathbf{k}_{3}+\mathbf{k}_{2}-\mathbf{k}_{1} \text { for } q=11(d=10) \\
\mathbf{k}_{9} & =\mathbf{k}_{5}-\mathbf{k}_{1}, \mathbf{k}_{10}=\mathbf{k}_{6}-\mathbf{k}_{2}, \mathbf{k}_{11}=\mathbf{k}_{7}-\mathbf{k}_{3}, \mathbf{k}_{12}=\mathbf{k}_{8}-\mathbf{k}_{4} \text { for } q=12, \quad(d=8) .
\end{aligned}
$$

Then $m_{s}^{*}:=\mathfrak{d} r_{s}=\sum_{j=1}^{2 q} m_{j} \beta_{j s} \in \mathbb{Z}$ and

$$
\mathbf{k}=\mathfrak{d}^{-1} \sum_{s=1}^{d} m_{s}^{*} \mathbf{k}_{s}^{*}:=\mathbf{k}\left(\mathbf{m}^{*}\right)
$$


where $\mathbf{m}^{*}:=\left(m_{1}^{*}, \ldots, m_{d}^{*}\right)$ and we define the following norm in the lattice $\Gamma$, identified with a subset of $\mathbb{Z}^{d}$ :

$$
N_{\mathbf{k}}:=\sum_{s=1}^{d}\left|m_{s}^{*}\right| .
$$

Remark 8. If $\mathfrak{d}=1$ we can identify $\Gamma$ with $\mathbb{Z}^{d}$. If $\mathfrak{d}>1$, for an arbitrary $\mathbf{m}^{*} \in \mathbb{Z}^{d} \backslash\{0\}$, we don't know a priori if there exists $\mathbf{k} \in \Gamma$ such that $\mathbf{k}\left(\mathbf{m}^{*}\right)=\mathbf{k}$.

In what follows we use Sobolev spaces defined as

$$
\mathcal{H}_{s}=\left\{W=\sum_{\mathbf{k} \in \Gamma} W^{(\mathbf{k})} e^{i \mathbf{k} \cdot \mathbf{x}} ;\|W\|_{s}^{2}=\sum_{\mathbf{k} \in \Gamma}\left(1+N_{\mathbf{k}}^{2}\right)^{s}\left|W^{(\mathbf{k})}\right|^{2}<\infty\right\},
$$

which are Hilbert spaces with the scalar product

$$
\langle W, V\rangle_{s}=\sum_{\mathbf{k} \in \Gamma}\left(1+N_{\mathbf{k}}^{2}\right)^{s} W^{(\mathbf{k})} \bar{V}^{(\mathbf{k})} .
$$

Definition 9. Let $N>1$ be an integer. We define the projection $\Pi_{N}$ acting in $\mathcal{H}_{s}$ : for $V \in \mathcal{H}_{s}$

$$
V=\sum_{\mathbf{k} \in \Gamma} V^{(\mathbf{k})} e^{i \mathbf{k} \cdot \mathbf{x}} \in \mathcal{H}_{s}, \quad \Pi_{N} V:=\sum_{\mathbf{k} \in \Gamma, N_{\mathbf{k}} \leq N} V^{(\mathbf{k})} e^{i \mathbf{k} \cdot \mathbf{x}} .
$$

The two following Lemmas are similar to classical results on Sobolev spaces.

Lemma 10. [BIS][lemma 5] Assume $q \geq 4$, then for $s>d / 2$, for any $U \in \mathcal{H}_{s}$ and any $V \in \mathcal{H}_{0}$, we have

$$
\|U V\|_{0} \leq c_{s}\|U\|_{s}\|V\|_{0}
$$

for a certain constant $c_{s}>0$.

Lemma 11. [BIS][lemma 6] (Moser-Nirenberg inequality) Assume $q \geq 4$, and let $s \geq s^{\prime}>$ $d / 2$ and let $U, V \in \mathcal{H}_{s}$. Then,

$$
\|U V\|_{s} \leq C\left(s, s^{\prime}\right)\left(\|U\|_{s}\|V\|_{s^{\prime}}+\|U\|_{s^{\prime}}\|V\|_{s}\right)
$$

for some positive constant $C\left(s, s^{\prime}\right)$ that depends only on $s$ and $s^{\prime}$. For $\ell \geq 0$ and $s>\ell+d / 2$, $\mathcal{H}_{s}$ is continuously embedded into $\mathcal{C}^{\ell}$.

Let us conclude this subsection in giving precisions on the small divisors occurring when we need to invert the operator $(1+\Delta)^{2}$. It appears that the quantities

$$
\left(1-|\mathbf{k}|^{2}\right)^{2}, \mathbf{k} \in \Gamma
$$

occur in the denominator of the inverted terms. So we need to bound the inverse of these quantities when they are not 0 . We can show

Lemma 12. Assume $q \geq 4$, then for any $\mathbf{k} \in \Gamma$ such that $|\mathbf{k}| \neq 1$, i.e. $\mathbf{k} \neq \mathbf{k}_{j}, j=1, \ldots, 2 q$ the following estimate holds true

$$
\left.|| \mathbf{k}\right|^{2}-1 \mid \geq \frac{c}{\left(1+N_{\mathbf{k}}^{2}\right)^{l_{0}}},
$$

for a certain $c>0$ only depending on $q$. 
Proof. From (7) we obtain, as in [IR10]

$$
\mathfrak{d}^{2}\left(|\mathbf{k}|^{2}-1\right)=\left\langle\sum_{s=1}^{d} m_{s}^{*} \mathbf{k}_{s}^{*}, \sum_{s=1}^{d} m_{s}^{*} \mathbf{k}_{s}^{*}\right\rangle-\mathfrak{d}^{2}=q_{0}-\mathfrak{d}^{2}+q_{1} \omega+\ldots+q_{l_{0}} \omega^{l_{0}}
$$

where $\omega=2 \cos \pi / q$ and the integer coefficients $q_{j}$ are quadratic in $\mathbf{m}^{*}$. It is shown in [IR10] (see Lemma 2.1) that if it is not 0 , there exists $C>0$ such that the right hand side is bounded from below by

$$
\frac{C}{\left(\left|q_{0}-\mathfrak{d}^{2}\right|+\left|q_{1}\right|+\ldots+\left|q_{l_{0}}\right|\right)^{l_{0}}} .
$$

Now, since we have $\left|q_{j}\right| \leq c^{\prime} N_{\mathbf{k}}^{2}, j=1, . ., l_{0}$, it is easy to conclude to the existence of $c>0$ in estimate (12).

\subsection{Formal computation}

Let us look for formal solutions of the steady Swift-Hohenberg equation

$$
\lambda U-(1+\Delta)^{2} U-U^{3}=0,
$$

We characterize the functions of interest by their Fourier coefficients on the quasilattice $\Gamma$ generated by the $2 q$ equally spaced unit vectors $\mathbf{k}_{j}$ (see (6)):

$$
U(\mathbf{x})=\sum_{\mathbf{k} \in \Gamma} u^{(\mathbf{k})} e^{i \mathbf{k} \cdot \mathbf{x}}, \quad \mathbf{x}=\left(x_{1}, x_{2}\right) \in \mathbb{R}^{2} .
$$

We seek a non trivial solution, bifurcating from 0 , parameterized by $\epsilon$, and which is invariant under rotations by $\pi / q$. As it is shown for example in [IR10], a formal computation with identification of orders in $\epsilon$ leads to (classically, it is easier to formulate the bifurcating branch with the parameter $\epsilon$ instead of expanding $U$ in powers of $\sqrt{\lambda}$ )

$$
U\left(x_{1}, x_{2}\right)=\epsilon u_{0}\left(x_{1}, x_{2}\right)+\epsilon^{3} u_{1}\left(x_{1}, x_{2}\right)+\ldots \quad \lambda=\epsilon^{2} \lambda_{2}+\epsilon^{4} \lambda_{4}+\ldots
$$

and gives at order $\mathcal{O}(\epsilon)$

$$
0=(1+\Delta)^{2} u_{0}
$$

We take as our basic solution a quasipattern that is invariant under rotations by $\pi / q$ :

$$
u_{0}=\sum_{j=1}^{2 q} e^{i \mathbf{k}_{j} \cdot \mathbf{x}}
$$

At order $\mathcal{O}\left(\epsilon^{3}\right)$ we have

$$
\lambda_{2} u_{0}-u_{0}^{3}=(1+\Delta)^{2} u_{1} .
$$

In order to solve this equation for $u_{1}$, we must impose a solvability condition, namely that the coefficients of $e^{i \mathbf{k}_{j} \cdot \mathbf{x}}, j=1, \ldots, 2 q$ on the left hand side of this equation must be zero. Because of the invariance under rotations by $\pi / q$, it is sufficient to cancel the coefficient of $e^{i \mathbf{k}_{1} \cdot \mathbf{x}}$. For the computation of the coefficient, we need the following property 
Property (see the proof in [BIS] and a related argument in Remark 28): If we have

$$
\mathbf{k}_{j}+\mathbf{k}_{l}+\mathbf{k}_{r}+\mathbf{k}_{s}=0 \text { for } j, l, r, s \in\{1,2 q\}
$$

then either $\mathbf{k}_{j}+\mathbf{k}_{l}=0$, or $\mathbf{k}_{j}+\mathbf{k}_{r}$, or $\mathbf{k}_{j}+\mathbf{k}_{s}=0$ (there are two pairs of opposite unit vectors).

This yields

$$
\lambda_{2}=3(2 q-1)
$$

which is strictly positive. Moreover we fix the uniqueness of functions $u_{n}, n \geq 1$ by imposing the orthogonality of $u_{n}$ with $u_{0}$. This also fixes the relationship between $\epsilon$ and $\lambda$. Hence

$$
\begin{aligned}
u_{1} & =\sum_{\mathbf{k} \in \Gamma,|\mathbf{k}| \neq 1} \alpha_{\mathbf{k}} e^{i \mathbf{k} \cdot \mathbf{x}}, \alpha_{\mathbf{k}}=0 \text { for } \mathbf{k} \neq \mathbf{k}_{j}+\mathbf{k}_{l}+\mathbf{k}_{r}, \text { and } \\
\alpha_{3 \mathbf{k}_{j}} & =-1 / 64, \quad \alpha_{2 \mathbf{k}_{j}+\mathbf{k}_{l}}=-\frac{3}{\left(1-\left|2 \mathbf{k}_{j}+\mathbf{k}_{l}\right|^{2}\right)^{2}}, \quad \mathbf{k}_{j}+\mathbf{k}_{l} \neq 0, \\
\alpha_{\mathbf{k}_{j}+\mathbf{k}_{l}+\mathbf{k}_{r}} & =-\frac{6}{\left(1-\left|\mathbf{k}_{j}+\mathbf{k}_{l}+\mathbf{k}_{r}\right|^{2}\right)^{2}}, j \neq l \neq r \neq j, \\
\mathbf{k}_{j}+\mathbf{k}_{l} & \neq 0, \quad \mathbf{k}_{j}+\mathbf{k}_{r} \neq 0, \quad \mathbf{k}_{r}+\mathbf{k}_{l} \neq 0 .
\end{aligned}
$$

We notice that for any $\mathbf{k}, \alpha_{\mathbf{k}}<0$ in $u_{1}$. At order $\mathcal{O}\left(\epsilon^{5}\right)$ we have

$$
\lambda_{4} u_{0}+\lambda_{2} u_{1}-3 u_{0}^{2} u_{1}=(1+\Delta)^{2} u_{2} .
$$

The solvability condition gives $\lambda_{4}$ equal to the coefficient of $e^{i \mathbf{k}_{1} \cdot \mathbf{x}}$ in $3 u_{0}^{2} u_{1}$, hence $\lambda_{4}<0$ (see [BIS] [section 2.2]).

It is shown in [IR10] that the series (14) is of Gevrey type. Moreover it is shown that a Borel resummation of this series, provides a quasi-periodic function which is solution of equation (2) up to an exponentially small term with respect to the bifurcation parameter $\epsilon$. We indeed wish a better result, i.e. the proof of the existence of a solution having the above asymptotic expansion (14) at the origin.

\subsection{Formulation of the problem}

Let us define the new unknown function $W$ in rewriting (14) as:

$$
\begin{aligned}
U & =U_{\epsilon}+\epsilon^{2} W, \\
U_{\epsilon} & =\epsilon u_{0}+\epsilon^{3} u_{1} \\
\lambda & =\epsilon^{2} \lambda_{2}-\epsilon^{3} \lambda^{\prime}
\end{aligned}
$$

where $u_{0}, u_{1}, \lambda_{2}$ are as above. Given a particular value of $\left(\lambda, \lambda^{\prime}\right), \lambda>0$ small enough and $\lambda^{\prime} \in\left[-\lambda_{0}, \lambda_{0}\right]$, we get $\epsilon$ by the implicit function theorem, and since $\lambda_{2}>0$, we obtain a unique positive $\epsilon$. All the corrections are in $W$. The aim is to show that the quasi-periodic function $W$ exists and is small as $\epsilon$ tends towards 0 . By construction we have

$$
(1+\Delta)^{2} U_{\epsilon}-\epsilon^{2} \lambda_{2} U_{\epsilon}+U_{\epsilon}^{3}=: \epsilon^{5} f_{\epsilon}
$$


with

$$
f_{\epsilon}=-\lambda_{2} u_{1}+3 u_{0}^{2} u_{1}+3 \epsilon^{2} u_{0} u_{1}^{2}+\epsilon^{4} u_{1}^{3},
$$

where $f_{\epsilon}$ is quasi-periodic, of order $\mathcal{O}(1)$ with a finite Fourier expansion, and is function of $\epsilon^{2}$.

Definition 13. Let us define the orthogonal projections $\Pi_{0}$ on $\left\{u_{0}\right\}^{\perp}$ and $\Pi_{1}=\mathbb{I}-\Pi_{0}$.

Applying $\Pi_{0}$ just consists in cancelling the terms with $|\mathbf{k}|=1$ in the Fourier expansion of $W \in \mathcal{H}_{s}$. This projection is orthogonal in any $\mathcal{H}_{s}, s \geq 0$.

After substituting (21) into the PDE (13), and dividing by $\epsilon^{2}$, we obtain an equation of the form

$$
F\left(\epsilon, \lambda^{\prime}, W\right):=L_{\epsilon, \lambda^{\prime}} W+\epsilon^{3} f_{\epsilon}+\lambda^{\prime} \epsilon U_{\epsilon}+3 \epsilon^{2} U_{\epsilon} W^{2}+\epsilon^{4} W^{3}=0,
$$

where

$$
\begin{aligned}
L_{\epsilon, \lambda^{\prime}} u & :=\left[(1+\Delta)^{2}-\epsilon^{2} \lambda_{2}+\epsilon^{3} \lambda^{\prime}\right] u+3 U_{\epsilon}^{2} u, \\
L_{\epsilon, \lambda^{\prime}, W} u & :=D_{W} F\left(\epsilon, \lambda^{\prime}, W\right) u=L_{\epsilon, \lambda^{\prime}} u+6 \epsilon^{2} U_{\epsilon} W u+3 \epsilon^{4} W^{2} u .
\end{aligned}
$$

We notice that

$$
\begin{aligned}
(1+\Delta)^{2} V & =\sum_{\mathbf{k} \in \Gamma}\left(1-|\mathbf{k}|^{2}\right)^{2} V^{(\mathbf{k})} e^{i \mathbf{k} \cdot \mathbf{x}} \\
|\mathbf{k}| & \leq \mathfrak{d}^{-1} N_{\mathbf{k}} \leq N_{\mathbf{k}}
\end{aligned}
$$

hence

$$
\left(1-|\mathbf{k}|^{2}\right)^{2} \leq\left(1+N_{\mathbf{k}}^{2}\right)^{2},
$$

so, the linear operator $(1+\Delta)^{2}$ is bounded from $\Pi_{0} \mathcal{H}_{r}$ to $\Pi_{0} \mathcal{H}_{r-4}$ for $r \geq 4$.

It is clear (see Lemma 10) that for a fixed $W \in \mathcal{H}_{s}, s>d / 2$, the operator $L_{\epsilon, \lambda^{\prime}, W}$ is an operator acting from any $\mathcal{H}_{r}, r \geq 4$, to $\mathcal{H}_{r-4}$ being uniformly bounded in $\epsilon, \lambda^{\prime}$, for $\epsilon \leq \epsilon_{0}, \lambda^{\prime} \in[-1,1]$

The operator $L_{\epsilon, \lambda^{\prime}, W}$ has not a bounded inverse, due to the small divisor problem mentioned in section 1.1. Notice however that we have from (12) the estimate

$$
\frac{1}{\left(|\mathbf{k}|^{2}-1\right)^{2}} \leq c_{0}\left(1+N_{\mathbf{k}}^{2}\right)^{2 l_{0}}
$$

which gives an upper bound of $\left[\widetilde{L_{0}}\right]^{-1}$, where $L_{0}:=L_{0, \lambda^{\prime}, W}$ and the superscript ${ }^{\sim}$ means the pseudoinverse acting from $\Pi_{0} \mathcal{H}_{r}=\mathcal{H}_{r} \cap\left\{u_{0}\right\}^{\perp}$ into $\Pi_{0} \mathcal{H}_{r-4 l_{0}}$.

In formal computations, we may choose $\lambda^{\prime}=-\epsilon \lambda_{4}+O\left(\epsilon^{3}\right)$ so that $W=\epsilon^{3} u_{2}+$ $O\left(\epsilon^{5}\right)\left\{u_{0}\right\}^{\perp}$. The introduction of $\lambda^{\prime} \neq 0$ modifies the definition of $\epsilon$ as the parameter describing the bifurcating solution curve. For an arbitrary $\lambda^{\prime}$ in $[-1,1]$, it results that $W$ is no longer in $\left\{u_{0}\right\}^{\perp}$, having now a component along $u_{0}$ depending on $\lambda^{\prime}$.

Let us decompose $W \in \mathcal{H}_{s}$ as (see Remark 15 below):

$$
W=\nu u_{0}+3 \nu \epsilon^{2} u_{1}+\epsilon^{2} V, \quad V \in\left\{u_{0}\right\}^{\perp} .
$$


Equation (23) decomposes into the bifurcation equation (projection onto the kernel of $(1+$ $\left.\Delta)^{2}\right)$ :

$$
\begin{aligned}
0= & \left(-\lambda_{2}+\epsilon \lambda^{\prime}\right) \nu u_{0}+\lambda^{\prime} u_{0}+\epsilon \Pi_{1} f_{\epsilon}+3 \Pi_{1}\left[\epsilon^{-2} U_{\epsilon}^{2}\left(\nu u_{0}+3 \nu \epsilon^{2} u_{1}+\epsilon^{2} V\right)\right]+ \\
& +3 \Pi_{1} U_{\epsilon}\left(\nu u_{0}+3 \nu \epsilon^{2} u_{1}+\epsilon^{2} V\right)^{2}+\epsilon^{2} \Pi_{1}\left(\nu u_{0}+3 \nu \epsilon^{2} u_{1}+\epsilon^{2} V\right)^{3},
\end{aligned}
$$

and the range equation (projection onto $\left\{u_{0}\right\}^{\perp}$ ):

$$
\begin{aligned}
0= & {\left[(1+\Delta)^{2}-\epsilon^{2} \lambda_{2}+\epsilon^{3} \lambda^{\prime}\right]\left(3 \nu u_{1}+V\right)+\epsilon \Pi_{0} f_{\epsilon}+\lambda^{\prime} \epsilon^{2} u_{1}+3 \Pi_{0}\left[\epsilon^{-2} U_{\epsilon}^{2}\left(\nu u_{0}+3 \nu \epsilon^{2} u_{1}+\epsilon^{2} V\right)\right]+} \\
& +3 \Pi_{0}\left[U_{\epsilon}\left(\nu u_{0}+3 \nu \epsilon^{2} u_{1}+\epsilon^{2} V\right)^{2}\right]+\epsilon^{2} \Pi_{0}\left[\left(\nu u_{0}+3 \nu \epsilon^{2} u_{1}+\epsilon^{2} V\right)^{3}\right] .
\end{aligned}
$$

After using the identities

$$
\Pi_{1} u_{0}^{3}=\lambda_{2} u_{0}, \quad 3 \Pi_{1} u_{0}^{2} u_{1}=\lambda_{4} u_{0}, \quad(1+\Delta)^{2} u_{1}+\Pi_{0} u_{0}^{3}=0,
$$

and from the expression (22) of $f_{\epsilon},(29)$ and (30) become

$$
\begin{gathered}
{\left[2 \nu \lambda_{2}+\epsilon \lambda_{4}+\lambda^{\prime}\right] u_{0}+\widetilde{f}(\epsilon, \nu)+\epsilon^{2} \Pi_{1}\left[f_{1}(\epsilon, \nu) V\right]+\epsilon^{5} \Pi_{1}\left[f_{2}(\epsilon, \nu) V^{2}\right]+\epsilon^{8} \Pi_{1}\left[f_{3}(\epsilon, \nu) V^{3}\right]=0} \\
\mathcal{F}\left(\epsilon, \lambda^{\prime}, \nu, V\right)=0
\end{gathered}
$$

Here, we have set

$$
\mathcal{F}\left(\epsilon, \lambda^{\prime}, \nu, V\right):=\mathcal{L}_{\epsilon, \lambda^{\prime}, \nu} V+g\left(\epsilon, \lambda^{\prime}, \nu\right)+3 \epsilon^{4} \Pi_{0}\left(U_{\epsilon} V^{2}\right)+\epsilon^{6} \Pi_{0}\left[3 \nu\left(u_{0}+3 \epsilon^{2} u_{1}\right) V^{2}+\epsilon^{2} V^{3}\right],
$$

where the linear operator $\mathcal{L}_{\epsilon, \lambda^{\prime}, \nu}$ is defined by

$$
\begin{aligned}
\mathcal{L}_{\epsilon, \lambda^{\prime}, \nu} V:=\quad & {\left[(1+\Delta)^{2}-\epsilon^{2} \lambda_{2}+\epsilon^{3} \lambda^{\prime}\right] V+3 \Pi_{0}\left(U_{\epsilon}^{2} V\right)+} \\
& +6 \nu \epsilon^{2} \Pi_{0}\left(U_{\epsilon}\left(u_{0}+3 \epsilon^{2} u_{1}\right) V\right)+3 \epsilon^{4} \nu^{2} \Pi_{0}\left[\left(u_{0}+3 \epsilon^{2} u_{1}\right)^{2} V\right],
\end{aligned}
$$

and

$$
\begin{aligned}
& g\left(\epsilon, \lambda^{\prime}, \nu\right):=\quad \epsilon^{2} \lambda^{\prime}(1+3 \epsilon \nu) u_{1}+\epsilon \Pi_{0} f_{\epsilon}+3 \nu \epsilon^{2} \Pi_{0}\left[\left(2 u_{0} u_{1}+\epsilon^{2} u_{1}^{2}\right) u_{0}\right]-3 \nu \epsilon^{2} \lambda_{2} u_{1}+ \\
& +9 \nu \Pi_{0}\left(U_{\epsilon}^{2} u_{1}\right)+3 \nu^{2} \Pi_{0}\left[U_{\epsilon}\left(u_{0}+3 \epsilon^{2} u_{1}\right)^{2}\right]+\epsilon^{2} \nu^{3} \Pi_{0}\left[\left(u_{0}+3 \epsilon^{2} u_{1}\right)^{3}\right] \\
& \widetilde{f}(\epsilon, \nu):=\left(\nu^{2} \epsilon \lambda_{2}+\nu \epsilon^{2}(4+3 \nu \epsilon) \lambda_{4}\right) u_{0}+3 \epsilon^{3}(1+3 \nu \epsilon)^{2} \Pi_{1}\left(u_{0} u_{1}^{2}\right)+\epsilon^{5}(1+\nu \epsilon)^{-1}(1+3 \nu \epsilon)^{3} \Pi_{1}\left(u_{1}^{3}\right) \\
& f_{1}(\epsilon, \nu):=(1+\nu \epsilon)^{-1}\left[3 U_{\epsilon}^{2}+6 \nu \epsilon^{2} U_{\epsilon}\left(u_{0}+3 \epsilon^{2} u_{1}\right)+3 \nu^{2} \epsilon^{4}\left(u_{0}+3 \epsilon^{2} u_{1}\right)^{2}\right], \\
& f_{2}(\epsilon, \nu):=3(1+\nu \epsilon)^{-1}\left[\epsilon^{-1} U_{\epsilon}+\nu \epsilon\left(u_{0}+3 \epsilon^{2} u_{1}\right)\right], \\
& f_{3}(\epsilon, \nu):=(1+\nu \epsilon)^{-1} \text {. }
\end{aligned}
$$

are analytic in their arguments $\left(\epsilon, \lambda^{\prime}, \nu\right) \in\left[0, \epsilon_{0}\right] \times[-1,1] \times\left[-\epsilon_{0}, \epsilon_{0}\right]$ for $\epsilon_{0}$ small enough. We notice that $g\left(\epsilon, \lambda^{\prime}, \nu\right) \in \mathcal{H}_{s}$ for any $s \geq 0$ and has a finite Fourier expansion. 
Remark 14. Even though the above computations are elementary, let us give more details for obtaining $\widetilde{f}(\epsilon, \nu)$ which corresponds to the part independent of $V$ in equation (29), divided by $(1+\nu \epsilon)$ :

$$
\begin{aligned}
& (1+\nu \epsilon)\left(\left[2 \nu \lambda_{2}+\epsilon \lambda_{4}+\lambda^{\prime}\right] u_{0}+\widetilde{f}(\epsilon, \nu)\right) \\
= & \left(-\lambda_{2}+\epsilon \lambda^{\prime}\right) \nu u_{0}+\lambda^{\prime} u_{0}+\epsilon \Pi_{1} f_{\epsilon}+3 \Pi_{1}\left[\epsilon^{-2} U_{\epsilon}^{2}\left(\nu u_{0}+3 \nu \epsilon^{2} u_{1}\right)\right]+ \\
& +3 \Pi_{1} U_{\epsilon}\left(\nu u_{0}+3 \nu \epsilon^{2} u_{1}\right)^{2}+\epsilon^{2} \Pi_{1}\left(\nu u_{0}+3 \nu \epsilon^{2} u_{1}\right)^{3} \\
= & (1+\nu \epsilon) \lambda^{\prime} u_{0}-\nu \lambda_{2} u_{0}+\epsilon \lambda_{4} u_{0}+\epsilon \Pi_{1}\left(3 \epsilon^{2} u_{0} u_{1}^{2}+\epsilon^{4} u_{1}^{3}\right)+3 \nu \lambda_{2} u_{0}+5 \nu \epsilon^{2} \lambda_{4}+ \\
& +21 \nu \epsilon^{4} \Pi_{1} u_{0} u_{1}^{2}+9 \nu \epsilon^{6} \Pi_{1} u_{1}^{3}+3 \nu^{2} \epsilon \lambda_{2}+7 \nu^{2} \epsilon^{3} \lambda_{4}+45 \nu^{2} \epsilon^{5} \Pi_{1} u_{0} u_{1}^{2}+ \\
& +27 \nu^{2} \epsilon^{7} \Pi_{1} u_{1}^{3}+\nu^{3} \epsilon^{2} \lambda_{2}+3 \nu^{3} \epsilon^{4} \lambda_{4}+27 \epsilon^{6} \nu^{3} \Pi_{1} u_{0} u_{1}^{2}+27 \epsilon^{8} \nu^{3} \Pi_{1} u_{1}^{3} \\
= & (1+\nu \epsilon)\left[\lambda^{\prime}+\left(2 \nu+\nu^{2} \epsilon\right) \lambda_{2}+\left(\epsilon+4 \nu \epsilon^{2}+3 \nu^{2} \epsilon^{3}\right) \lambda_{4}\right] u_{0}+ \\
& +(1+\nu \epsilon) 3 \epsilon^{3}(1+3 \nu \epsilon)^{2} \Pi_{1} u_{0} u_{1}^{2}+\epsilon^{5}(1+3 \nu \epsilon)^{3} \Pi_{1} u_{1}^{3} .
\end{aligned}
$$

Remark 15. We notice that the choice to add the term $3 \nu \epsilon^{2} u_{1}$ in the decomposition of $W$ in (28), allows to define a function $g\left(\epsilon, \lambda^{\prime}, \nu\right)$ such that

$$
g\left(0, \lambda^{\prime}, \nu\right)=0 .
$$

This is due to the fact that $3 \nu \Pi_{1}\left[\epsilon^{-2} U_{\epsilon}^{2} u_{0}\right]+(1+\Delta)^{2}\left(3 \nu u_{1}\right)=3 \nu \epsilon^{2} \Pi_{0}\left[\left(2 u_{0} u_{1}+\epsilon^{2} u_{1}^{2}\right) u_{0}\right]$.

We expect to find, for any $0<\epsilon \leq \epsilon_{0}$, a small eligible $\lambda^{\prime}$, with a solution $V$ of (32), function of $\left(\epsilon, \lambda^{\prime}, \nu\right)$ of order $O(|\nu|+\epsilon)$. Then putting $V\left(\epsilon, \lambda^{\prime}, \nu\right)$ into the bifurcation equation (31), we expect to find, $\lambda^{\prime}$ of order $\epsilon$ for any $a=\nu / \lambda^{\prime}$ in the small interval $\left[-\epsilon_{0}, \epsilon_{0}\right]$, which would lead to $W$ solution of (23).

\subsection{Strategy}

The main task is to solve (32) with respect to $V$. The lower bound (12) shows that the inverse of $\mathcal{L}_{0, \lambda^{\prime}, \nu}$ is an unbounded operator in $\Pi_{0} \mathcal{H}_{s}$, only bounded from $\Pi_{0} \mathcal{H}_{s}$ to $\Pi_{0} \mathcal{H}_{s-4 l_{0}}$. In other words, 0 belongs to the continuous spectrum of $\mathcal{L}_{0, \lambda^{\prime}, \nu}$. We then intend to use a Nash-Moser scheme.

Let us denote by $\mathcal{L}_{\epsilon, \lambda^{\prime}, \nu, V}$ the differential with respect to $V$ of $\mathcal{F}\left(\epsilon, \lambda^{\prime}, \nu, V\right)$ defined in (32), then the main difficulty to be solved below is to find a suitable bound for the inverse $\mathcal{L}_{\epsilon, \lambda^{\prime}, \nu, V}^{-1}$ for small values of $\epsilon$. Notice that $\mathcal{L}_{\epsilon, \lambda^{\prime}, \nu, V}$ is selfadjoint in $\Pi_{0} \mathcal{H}_{0}$ but not in $\Pi_{0} \mathcal{H}_{s}$ for $s>0$. This is due to the fact that the operator "multiplication by a function" is not self adjoint in $\mathcal{H}_{s}, s>0$. It is tempting to work on the small (real) eigenvalues to obtain a bound of its inverse. However, we are in an infinite dimensional space, the set of eigenvalues is dense on $\mathbb{R}^{+}$, and the spectrum does not contain only eigenvalues, since it is a closed subset of $\mathbb{R}$. This does not allow to use standard perturbation theory.

A reduction method, currently used in PDE systems, such as in water waves problems for example [IPT05], [IP09], [IP11], [AB15], is to use a change of coordinates which transforms the linear operator (here $\mathcal{L}_{\epsilon, \lambda^{\prime}, \nu, V}$ ) into a diagonal operator, plus a small regularizing perturbation, such that the inversion is made via a Neumann series. It seems that this method is unpracticable here due to the ellipticity of $\mathcal{L}_{0, \lambda^{\prime}, \nu}$.

The option we choose is to truncate the space to functions with finite Fourier expansions (with $\mathbf{k}$ such that $\left.N_{\mathbf{k}} \leq N\right)$. Then we solve the system (32) with respect to $V$ for $\left(\epsilon, \lambda^{\prime}, \nu\right) \in$ 
$\left(0, \epsilon_{1}\right) \times[-1,1] \times\left[-\epsilon_{0}, \epsilon_{0}\right]$ where $\epsilon_{1}<\epsilon_{0}$ are small enough, and $\lambda^{\prime}$ suitably chosen for any fixed $\epsilon$. Using good separation properties of the spectrum of $(1+\Delta)^{2}$, we use the version of the Nash-Moser implicit function theorem developped by Berti-Bolle in [BB10], BertiBolle-Procesi in [BBP10], and Berti, Corsi and Procesi in [BCP]. This is the object of the rest of section 2 , and of sections 3 and 4 .

Definition 16. Let $s \geq 0$ and let $N>1$ be an integer, we define

$$
E_{N}:=\Pi_{N} \Pi_{0} \mathcal{H}_{s}
$$

which consists in keeping in the Fourier expansion of $V \in \Pi_{0} \mathcal{H}_{s}$ only those $\mathbf{k} \in \Gamma$ such that $N_{\mathbf{k}} \leq N$.

\subsection{Tame properties}

In all what follows in the paper, we use the following classical notation for the Banach space of bounded linear operators from the Banach space $E$ into the Banach space $F$ :

$$
\mathcal{L}(E, F), \mathcal{L}(E) \text { if } E \equiv F \text {. }
$$

In the case where $E=\Pi_{0} \mathcal{H}_{r}$, we use the notation $\|\cdot\|_{r}$ for the norm in $\mathcal{L}\left(\Pi_{0} \mathcal{H}_{r}\right)$, as for the norm in $\Pi_{0} \mathcal{H}_{r}$, if there is no possible confusion.

Let us consider (32)

$$
\mathcal{F}\left(\epsilon, \lambda^{\prime}, \nu, V\right)=0,
$$

then, due to $(11)$ and to $(26), \mathcal{F}$ is analytic from $\left[0, \epsilon_{0}\right] \times[-1,1] \times\left[-\epsilon_{0}, \epsilon_{0}\right] \times \Pi_{0} \mathcal{H}_{r}$ to $\Pi_{0} \mathcal{H}_{r-4}$, for $r>d / 2$. Moreover for $v \in \Pi_{0} \mathcal{H}_{r}, r>d / 2$ let us define

$$
\mathcal{L}_{\epsilon, \lambda^{\prime}, \nu, V} v:=D_{V} \mathcal{F}\left(\epsilon, \lambda^{\prime}, \nu, V\right) v
$$

Hence

$$
\mathcal{L}_{\epsilon, \lambda^{\prime}, \nu, V} v=\mathcal{L}_{\epsilon, \lambda^{\prime}, \nu} v+\epsilon^{5} \mathcal{M}(\epsilon, \nu, V) v,
$$

where $\mathcal{L}_{\epsilon, \lambda^{\prime}, \nu}$ is defined in (34), and where

$$
\mathcal{M}(\epsilon, \nu, V) v:=6 \Pi_{0}\left(\epsilon^{-1} U_{\epsilon} V v\right)+6 \nu \epsilon \Pi_{0}\left[\left(u_{0}+3 \epsilon^{2} u_{1}\right) V v\right]+3 \epsilon^{3} \Pi_{0}\left(V^{2} v\right) .
$$

We have the following decomposition

$$
\mathcal{L}_{\epsilon, \lambda^{\prime}, \nu, V} v=\mathcal{L}_{0} v+\epsilon^{2} T\left(\epsilon, \lambda^{\prime}, \nu, V\right) v
$$

with the "diagonal" operator $\mathcal{L}_{0}$ defined by

$$
\mathcal{L}_{0}=\Pi_{0}(1+\Delta)^{2},
$$

and

$$
\begin{aligned}
\epsilon^{2} T\left(\epsilon, \lambda^{\prime}, \nu, V\right) v:=\quad & \left(-\epsilon^{2} \lambda_{2}+\epsilon^{3} \lambda^{\prime}\right) v+3 \Pi_{0}\left(U_{\epsilon}^{2} v\right)+6 \nu \epsilon^{2} \Pi_{0}\left[U_{\epsilon}\left(u_{0}+3 \epsilon^{2} u_{1}\right) v\right] \\
& +3 \epsilon^{4} \nu^{2} \Pi_{0}\left[\left(u_{0}+3 \epsilon^{2} u_{1}\right)^{2} v\right]+\epsilon^{5} \mathcal{M}(\epsilon, \nu, V) v
\end{aligned}
$$


It follows from Lemma 10 that the operator $T\left(\epsilon, \lambda^{\prime}, \nu, V\right)$ is analytic in its arguments for $\left(\epsilon, \lambda^{\prime}, \nu, V\right) \in\left[0, \epsilon_{0}\right] \times[-1,1] \times\left[-\epsilon_{0}, \epsilon_{0}\right] \times \Pi_{0} \mathcal{H}_{s}, s \geq s_{0}>d / 2$, taking values in $\mathcal{L}\left(\Pi_{0} \mathcal{H}_{r}\right)$, for $0 \leq r \leq s$.

Let $s \geq s_{0}>d / 2$ and $\left(\epsilon, \lambda^{\prime}, \nu, V\right) \in\left[0, \epsilon_{0}\right] \times[-1,1] \times\left[-\epsilon_{0}, \epsilon_{0}\right] \times \Pi_{0} \mathcal{H}_{s},\|V\|_{s_{0}}<1$. Then we have

$$
\mathcal{F}\left(0, \lambda^{\prime}, \nu, 0\right)=0
$$

as well as

$$
\max \left(\left\|\partial_{\epsilon} \mathcal{F}\left(\epsilon, \lambda^{\prime}, \nu, V\right)\right\|\left\|_{s}, \epsilon^{-2}\right\| \partial_{\lambda^{\prime}} \mathcal{F}\left(\epsilon, \lambda^{\prime}, \nu, V\right)\left\|_{s}, \epsilon^{-2}\right\| \partial_{\nu} \mathcal{F}\left(\epsilon, \lambda^{\prime}, \nu, V\right) \|_{s}\right) \leq C(s)\left(1+\|V\|_{s}\right) .
$$

Using Lemma 11, we also obtain

$$
\begin{gathered}
\left\|\mathcal{L}_{\epsilon, \lambda^{\prime}, \nu, V} v\right\|_{s} \leq C(s)\left[\|v\|_{s+4}+\epsilon^{5}\|v\|_{s_{0}}\|V\|_{s}\right], \\
\left\|D_{V}^{2} \mathcal{F}\left(\epsilon, \lambda^{\prime}, \nu, V\right)(h, v)\right\|_{s} \leq C(s) \epsilon^{5}\left(\|h\|\left\|_{s}\right\| v\left\|_{s_{0}}+\right\| h\|\|_{s_{0}}\|v\|_{s}+\|V\|\left\|_{s}\right\| h\|\|_{s_{0}}\|v\|_{s_{0}}\right), \\
\epsilon^{-1}\left\|\partial_{\epsilon} \mathcal{L}_{\epsilon, \lambda^{\prime}, \nu, V} v\right\|_{s}+\epsilon^{-3}\left\|\partial_{\lambda^{\prime}} \mathcal{L}_{\epsilon, \lambda^{\prime}, \nu, V} v\right\|_{s}+\epsilon^{-3}\left\|\partial_{\nu} \mathcal{L}_{\epsilon, \lambda^{\prime}, \nu, V} v\right\|_{s} \leq C(s)\left[\|v\|_{s}+\epsilon^{3}\|v\|\left\|_{s_{0}}\right\| V \|_{s}\right] .
\end{gathered}
$$

\subsection{Estimate of $\left(\Pi_{N} \Pi_{0} \mathcal{L}_{\epsilon, \lambda^{\prime}, \nu, V} \Pi_{N} \Pi_{0}\right)^{-1}$ in $\mathcal{H}_{s}$ for small $N$}

Lemma 17. Let $S>s_{0}>l_{0}+1$ and $\epsilon_{0}>0$ small enough. Then there exists $c_{2}>0$ with the following property. For $0<\epsilon \leq \epsilon_{0}$ let $N \leq M_{\epsilon}:=\left[\frac{c_{2}}{\epsilon^{1 / 2 l_{0}}}\right]$ where the brackets $[\cdot]$ mean "the integer part of", and $\left(\epsilon, \lambda^{\prime}, \nu, V\right) \in\left(0, \epsilon_{0}\right] \times[-1,1] \times\left[-\epsilon_{0}, \epsilon_{0}\right] \times E_{N}$. Then the following estimate holds true for $s \in\left[s_{0}, S\right]$ and $\|V\|_{s} \leq 1$

$$
\left\|\left(\Pi_{N} \Pi_{0} \mathcal{L}_{\epsilon, \lambda^{\prime}, \nu, V} \Pi_{N} \Pi_{0}\right)^{-1}\right\|_{s} \leq 2 c_{0}\left(1+N^{2}\right)^{2 l_{0}} .
$$

The same is valid for $s=0$ with $\|V\|_{s_{0}} \leq 1$.

Proof. From (27), it follows that the operator $(1+\Delta)^{2}$ has an inverse in $\Pi_{N} \Pi_{0} \mathcal{H}_{s}$ for any $s \geq 0$, bounded as

$$
\left\|\left(\Pi_{N} \Pi_{0}(1+\Delta)^{2} \Pi_{N} \Pi_{0}\right)^{-1}\right\|_{s} \leq c_{0}\left(1+N^{2}\right)^{2 l_{0}} .
$$

We can write

$$
\Pi_{N} \Pi_{0} \mathcal{L}_{\epsilon, \lambda^{\prime}, \nu, V} \Pi_{N} \Pi_{0}=\Pi_{N} \Pi_{0}(1+\Delta)^{2} \Pi_{N} \Pi_{0}+\epsilon^{2} \Pi_{N} \Pi_{0} T\left(\epsilon, \lambda^{\prime}, \nu, V\right) \Pi_{N} \Pi_{0},
$$

with

$$
\left\|T\left(\epsilon, \lambda^{\prime}, \nu, V\right) v\right\|_{s} \leq c(s)\left(\|v\|_{s}+\epsilon\|V\|_{s}\|v\|_{s_{0}}\right) .
$$

for $s \geq s_{0}$, and

$$
\left\|T\left(\epsilon, \lambda^{\prime}, \nu, V\right) v\right\|_{0} \leq c(0)\left(1+\epsilon\|V\|_{s_{0}}\right)\|v\|_{0} .
$$

for $s=0$. Moreover, for

$$
c_{0} c(s)(1+\epsilon) \epsilon^{2}\left(1+N^{2}\right)^{2 l_{0}}<1 / 2
$$

we can invert $\Pi_{N} \Pi_{0} \mathcal{L}_{\epsilon, \lambda^{\prime}, \nu, V} \Pi_{N} \Pi_{0}$ via Neumann series and obtain the estimate (41). It is clear that this estimate holds provided that $c_{2} \leq 2 c_{0} c(s)$ for all $s \in\left[s_{0}, S\right]$ and for $s=0$ provided that $\|V\|_{s_{0}} \leq 1$, so that

$$
N \leq M_{\epsilon}=\left[\frac{c_{2}}{\epsilon^{1 / 2 l_{0}}}\right] \leq\left\{\left[2 c_{0} c(s)(1+\epsilon) \epsilon^{2}\right]^{-1 / 2 l_{0}}-1\right\}^{1 / 2} .
$$




\subsection{Estimate of $\left(\Pi_{N} \Pi_{0} \mathcal{L}_{\epsilon, \lambda^{\prime}, \nu, V} \Pi_{N} \Pi_{0}\right)^{-1}$ in $\mathcal{H}_{0}$ for large $N$}

In this section $N$ is fixed. In the rest of the paper, we need to introduce the following subset $\Lambda$ of $\mathbb{R}^{2}$ :

$$
\Lambda=\left\{\left(\lambda^{\prime}, \nu\right) \in \mathbb{R}^{2} ;|\nu| \leq \epsilon_{0}\left|\lambda^{\prime}\right|,\left|\lambda^{\prime}\right| \leq 1\right\} .
$$

This is useful for the control of eigenvalues of the linearized operator. Let us now define for $M>0$ and $s_{0}>d / 2$

$$
\mathcal{U}_{M}^{(N)}:=\left\{u \in C ^ { 1 } \left(\left[0, \epsilon_{1}\left[\times \Lambda, E_{N}\right) ; u\left(0, \lambda^{\prime}, \nu\right)=0,\|u\|_{s_{0}} \leq 1,\left\|\partial_{\epsilon, \lambda^{\prime}, \nu} u\right\|_{s_{0}} \leq M\right\} .\right.\right.
$$

We may observe that (36) says that the selfadjoint linear operator $\mathcal{L}_{\epsilon, \lambda^{\prime}, \nu, V}$ takes the form

$$
\mathcal{L}_{\epsilon, \lambda^{\prime}, \nu, V}=\mathcal{L}_{\epsilon, \lambda^{\prime}, \nu}+\epsilon^{5} \mathcal{M}(\epsilon, \nu, V),
$$

where $\mathcal{L}_{\epsilon, \lambda^{\prime}, \nu}$ is given by (34), is analytic in its arguments and $\lambda^{\prime}$ only occurs as $\epsilon^{3} \lambda^{\prime} \mathbb{I}$.

Let us consider the full linear operator $\Pi_{N} \Pi_{0} \mathcal{L}_{\epsilon, \lambda^{\prime}, \nu, V\left(\epsilon, \lambda^{\prime}, \nu\right)} \Pi_{N} \Pi_{0}$ where $V \in \mathcal{U}_{M}^{(N)}$. Since this symmetric operator is $C^{1}$ in its arguments, then after a suitable numbering of its eigenvalues $\mu_{j}^{(N)}\left(\epsilon, \lambda^{\prime}, \nu\right)$ it results that these ones are $C^{1}$ functions of $\nu$ for $\left(\epsilon, \lambda^{\prime}\right)$ fixed and $\left(\lambda^{\prime}, \nu\right)$ in $\Lambda$ ([Kato] theorem 6.8 p.122). In any $\nu_{0}$, the derivative $\partial_{\nu} \mu_{j}^{(N)}\left(\epsilon, \lambda^{\prime}, \nu_{0}\right)$ of an eigenvalue $\mu_{j}^{(N)}\left(\epsilon, \lambda^{\prime}, \nu\right)$ is in fact an eigenvalue of the operator (see [Kato] theorem 6.8 p.122 and see formula (6.5) p.123)

$$
\left.P_{\nu_{0}} \Pi_{N} \Pi_{0} \partial_{\nu}\left[\mathcal{L}_{\epsilon, \lambda^{\prime}, \nu}+\epsilon^{5} \mathcal{M}\left(\epsilon, \nu, V\left(\epsilon, \lambda^{\prime}, \nu\right)\right)\right]\right|_{\nu=\nu_{0}} P_{\nu_{0}}=\epsilon^{3} O p_{1}+\epsilon^{4} O p_{2},
$$

with

$$
O p_{1}=: P_{\nu_{0}} \Pi_{N} \Pi_{0}\left[6 u_{0}^{2} \cdot\right] \Pi_{N} \Pi_{0} P_{\nu_{0}}, \quad O p_{2}=P_{\nu_{0}} \Pi_{N} \Pi_{0} O(1) \Pi_{N} \Pi_{0} P_{\nu_{0}}
$$

where $P_{\nu_{0}}$ is the orthogonal total projector corresponding to the eigenvalue $\mu_{j}^{(N)}\left(\epsilon, \lambda^{\prime}, \nu_{0}\right)$ assumed to be multiple (otherwise all is simpler), and where the operator $O p_{2}$ is uniformly bounded with respect to $\left(\lambda^{\prime}, \nu\right) \in \Lambda$ and also with respect to $N \in \mathbb{N}$. In the above formula the orthogonal projector $P_{\nu_{0}}$ depends on $N$ and is continuous with respect to $\left(\epsilon, \lambda^{\prime}, \nu_{0}\right)$, following (for example) the Theorem 5.1 in [Kato] p.107. Notice that the operator $O p_{1}+$ $\epsilon O p_{2}$ is selfadjoint and continuously dependent on $\left(\epsilon, \lambda^{\prime}, \nu_{0}\right)$. Hence, dividing by $\epsilon^{3}$ the eigenvalues of the operator (46), one can write

$$
\partial_{\nu} \mu_{j}^{(N)}\left(\epsilon, \lambda^{\prime}, \nu_{0}\right)=\epsilon^{3} \alpha_{j}^{(N)}\left(\epsilon, \lambda^{\prime}, \nu_{0}\right)
$$

where $\alpha_{j}^{(N)}\left(\epsilon, \lambda^{\prime}, \nu_{0}\right)$ depends continuously on $\left(\epsilon, \lambda^{\prime}, \nu_{0}\right)$ as this results from the general result on eigenvalues of operators depending continuously on their arguments, and it may be written as

$$
\alpha_{j}^{(N)}\left(\epsilon, \lambda^{\prime}, \nu_{0}\right)=\left\langle 6 u_{0}^{2} \zeta_{j}^{(N)}\left(\epsilon, \lambda^{\prime}, \nu_{0}\right), \zeta_{j}^{(N)}\left(\epsilon, \lambda^{\prime}, \nu_{0}\right)\right\rangle_{0}+\mathcal{O}(\epsilon)
$$

as explained below. In (49) $\zeta_{j}^{(N)}\left(\epsilon, \lambda^{\prime}, \nu_{0}\right)$ denotes some eigenvector, of norm 1, of the self adjoint operator $\Pi_{N} \Pi_{0} \mathcal{L}_{\epsilon, \lambda^{\prime}, \nu_{0}, V\left(\epsilon, \lambda^{\prime}, \nu_{0}\right)} \Pi_{N} \Pi_{0}$ in the range of $P_{\nu_{0}} \Pi_{N} \Pi_{0}$. Indeed, we can diagonalize the symmetric operator $O p_{1}$ in the range of $P_{\nu_{0}} \Pi_{N} \Pi_{0}$ spanned by eigenvectors 
belonging to the eigenvalue $\mu_{j}\left(\epsilon, \lambda^{\prime}, \nu_{0}\right)$ of $\Pi_{N} \Pi_{0} \mathcal{L}_{\epsilon, \lambda^{\prime}, \nu_{0}, V\left(\epsilon, \lambda^{\prime}, \nu_{0}\right)} \Pi_{N} \Pi_{0}$, so that any eigenvalue of $O p_{1}$ reads as

$$
\left\langle 6 P_{\nu_{0}} \Pi_{N} \Pi_{0} u_{0}^{2} \zeta_{j}^{(N)}\left(\epsilon, \lambda^{\prime}, \nu_{0}\right), \zeta_{j}^{(N)}\left(\epsilon, \lambda^{\prime}, \nu_{0}\right)\right\rangle_{0}=\left\langle 6 u_{0}^{2} \zeta_{j}^{(N)}\left(\epsilon, \lambda^{\prime}, \nu_{0}\right), \zeta_{j}^{(N)}\left(\epsilon, \lambda^{\prime}, \nu_{0}\right)\right\rangle_{0},
$$

since $\zeta_{j}^{(N)}\left(\epsilon, \lambda^{\prime}, \nu_{0}\right)$ is a suitable combination of vectors in the range mentioned above. Moreover, using the Lidskii theorem (see [Kato] p.125-126) the rest $\mathcal{O}(\epsilon)$ in (49) is uniformly bounded with respect to $\left(\lambda^{\prime}, \nu_{0}\right) \in \Lambda$ and also with respect to $N \in \mathbb{N}$, since $O p_{2}$ in (46), (47) is bounded independently of $N$ (projectors have norm 1 ).

Remark 18. It might be tempting to replace $P_{\nu_{0}}$ by $P_{0}$ which is defined as the orthogonal total projector corresponding to the limit projector as $\left(\lambda^{\prime}, \nu_{0}\right) \rightarrow(0,0)$ of the group of eigenvalues merging in $\mu_{j}\left(\epsilon, \lambda^{\prime}, \nu_{0}\right)$. We might obtain an apparently simpler formula than (48), (49). Then the problem will be the non uniform estimate with respect to $N$, and this is not good for what we make in Section 5.

Now consider the dependence of $\mu_{j}^{(N)}\left(\epsilon, \lambda^{\prime}, \nu\right)$ with respect to $\lambda^{\prime}$. Since the ordering of eigenvalues is now fixed, we cannot use again the argument above. However, the operator

$$
\mathcal{L}_{\epsilon, \lambda^{\prime}, \nu}^{\prime(N)}=: \Pi_{N} \Pi_{0} \mathcal{L}_{\epsilon, \lambda^{\prime}, \nu, V\left(\epsilon, \lambda^{\prime}, \nu\right)} \Pi_{N} \Pi_{0}-\epsilon^{3} \lambda^{\prime} \mathbb{I}
$$

is symmetric, it has eigenvalues $\mu_{j}^{(N)}\left(\epsilon, \lambda^{\prime}, \nu\right)-\epsilon^{3} \lambda^{\prime}$ and, thanks to (45) and (36) we have the estimate

$$
\left\|\mathcal{L}_{\epsilon, \lambda_{2}^{\prime}, \nu}^{(N)}-\mathcal{L}_{\epsilon, \lambda_{1}^{\prime}, \nu}^{(N)}\right\|_{0} \leq C^{\prime} \epsilon^{5}\left|\lambda_{2}^{\prime}-\lambda_{1}^{\prime}\right|
$$

where the dependence in $\lambda^{\prime}$ comes from $V\left(\epsilon, \lambda^{\prime}, \nu\right)$ in operator $\mathcal{M}$. It results from the Lidskii theorem ([Kato] p.125-126) that the difference between the eigenvalues of $\mathcal{L}_{\epsilon, \lambda_{2}^{\prime}, \nu}^{(N)}$ and $\mathcal{L}_{\epsilon, \lambda_{1}^{\prime}, \nu}^{\prime(N)}$ satisfies

$$
\left|\mu_{j_{2}}^{(N)}\left(\epsilon, \lambda_{2}^{\prime}, \nu\right)-\mu_{j_{1}}^{(N)}\left(\epsilon, \lambda_{1}^{\prime}, \nu\right)-\epsilon^{3}\left(\lambda_{2}^{\prime}-\lambda_{1}^{\prime}\right)\right| \leq C^{\prime} \epsilon^{5}\left|\lambda_{2}^{\prime}-\lambda_{1}^{\prime}\right|,
$$

where the numbering of eigenvalues might depend on $\lambda^{\prime}$. In fact if $\mu_{j_{1}}^{(N)}\left(\epsilon, \lambda_{1}^{\prime}, \nu\right)$ is a simple eigenvalue, we have $j_{1}=j_{2}$ for $\lambda_{2}^{\prime}$ close enough to $\lambda_{1}^{\prime}$, whereas if this eigenvalue is multiple we may have $j_{1} \neq j_{2}$. However, in this latter case, for $\lambda_{2}^{\prime}$ tending towards $\lambda_{1}^{\prime}$ we have a finite group of eigenvalues merging to $\mu_{j_{1}}^{(N)}\left(\epsilon, \lambda_{1}^{\prime}, \nu\right)$ for $\lambda_{2}^{\prime}=\lambda_{1}^{\prime}$, so that the numbering is such that

$$
\mu_{j_{2}}^{(N)}\left(\epsilon, \lambda_{1}^{\prime}, \nu\right)=\mu_{j_{1}}^{(N)}\left(\epsilon, \lambda_{1}^{\prime}, \nu\right) .
$$

Finally, in all cases we have

$$
\left|\mu_{j}^{(N)}\left(\epsilon, \lambda_{2}^{\prime}, \nu\right)-\mu_{j}^{(N)}\left(\epsilon, \lambda_{1}^{\prime}, \nu\right)-\epsilon^{3}\left(\lambda_{2}^{\prime}-\lambda_{1}^{\prime}\right)\right| \leq C^{\prime} \epsilon^{5}\left|\lambda_{2}^{\prime}-\lambda_{1}^{\prime}\right|,
$$

which implies, for $\lambda_{2}^{\prime}-\lambda_{1}^{\prime} \geq 0$,

$$
\left(\epsilon^{3}-C^{\prime} \epsilon^{5}\right)\left(\lambda_{2}^{\prime}-\lambda_{1}^{\prime}\right) \leq \mu_{j}^{(N)}\left(\epsilon, \lambda_{2}^{\prime}, \nu\right)-\mu_{j}^{(N)}\left(\epsilon, \lambda_{1}^{\prime}, \nu\right) \leq\left(\epsilon^{3}+C^{\prime} \epsilon^{5}\right)\left(\lambda_{2}^{\prime}-\lambda_{1}^{\prime}\right) .
$$

It is then clear that for $\epsilon \leq \epsilon_{1}$ small enough, the function $\lambda^{\prime} \mapsto \mu_{j}^{(N)}\left(\epsilon, \lambda^{\prime}, \nu\right)$ is strictly increasing in $\lambda^{\prime}$ for $\lambda^{\prime} \in[-1,1]$, with a slope larger than $\epsilon^{3} / 2$. 
Now for certain $\tau$ and $\gamma>0$ to be determined later, we define a "bad" set of $\lambda^{\prime}$ :

$$
B_{\epsilon, \gamma, \nu}^{(N)}(V)=\left\{\lambda^{\prime} \in[-1,1] ;\left(\lambda^{\prime}, \nu\right) \in \Lambda, \exists j \in\{1,2, \ldots, \mathcal{N}\},\left|\mu_{j}^{(N)}\left(\epsilon, \lambda^{\prime}, \nu\right)\right|<\frac{\gamma}{N^{\tau}}\right\} .
$$

Because of the above strict monotonicity in $\lambda^{\prime}$ this "bad" set is the union $\cup_{j=1}^{\mathcal{N}}\left(\lambda_{j}^{\prime-}(\epsilon, \nu), \lambda_{j}^{\prime+}(\epsilon, \nu)\right)$ of small intervals defined by

$$
\mu_{j}^{(N)}\left(\epsilon, \lambda_{j}^{\prime \pm}, \nu\right)=\frac{ \pm \gamma}{N^{\tau}} .
$$

Now the lower bound $\left(\epsilon^{3} / 2\right)$ of the slope shows that

$$
\epsilon^{3} / 2\left[\lambda_{j}^{\prime+}(\epsilon, \nu)-\lambda_{j}^{\prime-}(\epsilon, \nu)\right] \leq \frac{2 \gamma}{N^{\tau}},
$$

hence

$$
\left|\lambda_{j}^{\prime+}(\epsilon, \nu)-\lambda_{j}^{\prime-}(\epsilon, \nu)\right| \leq \frac{4 \gamma}{\epsilon^{3} N^{\tau}} .
$$

We define the "bad strip" of degree $N, B S_{N}(V)$, to be the set in the $\left(\nu, \lambda^{\prime}\right)$ plane :

$$
B S_{N}(V):=\left\{\left(\lambda^{\prime}, \nu\right) \in \Lambda ; \lambda^{\prime} \in\left(\lambda_{j}^{\prime-}(\epsilon, \nu), \lambda_{j}^{\prime+}(\epsilon, \nu)\right)\right\},
$$

for $j$ occurring in the definition of "bad" sets $B_{\epsilon, \gamma, \nu}^{(N)}(V)$. We notice that the dimension $\mathcal{N}$ of the space $\Pi_{N} \Pi_{0} \mathcal{H}_{s}$ is independent of $s$, and is bounded by $c_{1} N^{d}$ since $\mathfrak{d}^{-1} \mathbf{k}$ lies in a subset of $\mathbb{Z}^{d}$, bounded by $N$. Hence it is clear that for any fixed $\nu \in\left[-\epsilon_{0}, \epsilon_{0}\right]$, the measure of the bad set of $\lambda^{\prime}$ such that $\left(\lambda^{\prime}, \nu\right) \in \Lambda$, is bounded by $\frac{4 \gamma \mathcal{N}}{\epsilon^{3} N^{\tau}}$, so

$$
\text { meas }\left(B_{\epsilon, \gamma, \nu}^{(N)}(V)\right) \leq \frac{4 \gamma c_{1}}{\epsilon^{3} N^{\tau-d}}
$$

and a first condition to have a set of small measure for $N$ large, is to choose $\tau>d$.

Now, we need to specify the behavior of $\lambda_{j}^{\prime \pm}(\epsilon, \nu)$ in function of $\nu$, for having an idea of the form of the strips. We have by construction for $\nu_{1}, \nu_{2}$ in $\left(-\epsilon_{0}, \epsilon_{0}\right)$ :

$$
\mu_{j}^{(N)}\left(\epsilon, \lambda_{j}^{\prime+}\left(\epsilon, \nu_{2}\right), \nu_{2}\right)-\mu_{j}^{(N)}\left(\epsilon, \lambda_{j}^{\prime+}\left(\epsilon, \nu_{1}\right), \nu_{1}\right)=0,
$$

i.e.

$\mu_{j}^{(N)}\left(\epsilon, \lambda_{j}^{\prime+}\left(\epsilon, \nu_{2}\right), \nu_{2}\right)-\mu_{j}^{(N)}\left(\epsilon, \lambda_{j}^{\prime+}\left(\epsilon, \nu_{1}\right), \nu_{2}\right)=-\left[\mu_{j}^{(N)}\left(\epsilon, \lambda_{j}^{\prime+}\left(\epsilon, \nu_{1}\right), \nu_{2}\right)-\mu_{j}^{(N)}\left(\epsilon, \lambda_{j}^{\prime+}\left(\epsilon, \nu_{1}\right), \nu_{1}\right)\right]$.

Now, from (50) we have

$$
\mu_{j}^{(N)}\left(\epsilon, \lambda_{j}^{\prime+}\left(\epsilon, \nu_{2}\right), \nu_{2}\right)-\mu_{j}^{(N)}\left(\epsilon, \lambda_{j}^{\prime+}\left(\epsilon, \nu_{1}\right), \nu_{2}\right)=\left[\epsilon^{3}+\mathcal{O}\left(\epsilon^{5}\right)\right]\left(\lambda_{j}^{\prime+}\left(\epsilon, \nu_{2}\right)-\lambda_{j}^{\prime+}\left(\epsilon, \nu_{1}\right)\right),
$$

where the term $\mathcal{O}\left(\epsilon^{5}\right)$ is independent of $N$, and from (48), the continuity of the derivative and the mean value theorem, we have

$$
\mu_{j}\left(\epsilon, \lambda_{j}^{\prime+}\left(\epsilon, \nu_{1}\right), \nu_{2}\right)-\mu_{j}\left(\epsilon, \lambda_{j}^{\prime+}\left(\epsilon, \nu_{1}\right), \nu_{1}\right)=\epsilon^{3} \alpha_{j}^{(N)}\left(\epsilon, \lambda_{j}^{\prime+}\left(\epsilon, \nu_{1}\right), \nu^{\prime}\right)\left(\nu_{2}-\nu_{1}\right),
$$

where $\nu^{\prime} \in\left[\nu_{1}, \nu_{2}\right]$. Hence

$$
\left[1+\mathcal{O}\left(\epsilon^{2}\right)\right]\left(\lambda_{j}^{\prime+}\left(\epsilon, \nu_{2}\right)-\lambda_{j}^{\prime+}\left(\epsilon, \nu_{1}\right)\right)=-\alpha_{j}^{(N)}\left(\epsilon, \lambda_{j}^{\prime+}\left(\epsilon, \nu_{1}\right), \nu^{\prime}\right)\left(\nu_{2}-\nu_{1}\right),
$$


where the term bounded by $\mathcal{O}\left(\epsilon^{2}\right)$ depends on $\nu_{1}$ and $\nu_{2}$ but is bounded independently of $N$. Hence for $\epsilon$ small enough

$$
\lambda_{j}^{\prime+}\left(\epsilon, \nu_{2}\right)-\lambda_{j}^{\prime+}\left(\epsilon, \nu_{1}\right)=-\alpha_{j}^{(N)}\left(\epsilon, \lambda_{j}^{\prime+}\left(\epsilon, \nu_{1}\right), \nu^{\prime}\right)\left[1+\mathcal{O}\left(\epsilon^{2}\right)\right]\left(\nu_{2}-\nu_{1}\right) .
$$

The same analysis holds for $\lambda_{j}^{\prime-}$. Now, we shall see in section 5 that $\lambda^{\prime}$ and $\nu$ may vary on a very small set (at most of order $\epsilon$ ) hence the coefficients $\alpha_{j}^{(N)}\left(\epsilon, \lambda_{j}^{\prime \pm}\left(\epsilon, \nu_{1}\right), \nu^{\prime \pm}\right)$ do not vary much, and this means that, in the $\left(\nu, \lambda^{\prime}\right)$ plane, the bad strips are nearly rectilinear.

Let us define

$$
G_{\epsilon, \gamma, \nu}^{(N)}(V):=[-1,1] \backslash B_{\epsilon, \gamma, \nu}^{(N)}(V) .
$$

The following remark is crucial in what follows.

Remark 19. If $\lambda^{\prime} \in G_{\epsilon, \gamma, \nu}^{(N)}(V)$, then all eigenvalues of $\Pi_{0} \Pi_{N} \mathcal{L}_{\epsilon, \lambda^{\prime}, \nu, V\left(\epsilon, \lambda^{\prime}, \nu\right)} \Pi_{N} \Pi_{0}$ are at a distance $\geq \frac{\gamma}{N^{\tau}}$ from 0 .

From the property that $\Pi_{0} \Pi_{N} \mathcal{L}_{\epsilon, \lambda^{\prime}, \nu, V} \Pi_{N} \Pi_{0}$ is selfadjoint in $\Pi_{N} \Pi_{0} \mathcal{H}_{0}=E_{N}$, it results that we have

Lemma 20. For $V$ fixed in $\mathcal{U}_{M}^{(N)}$ and $\epsilon$ fixed $\in\left[0, \epsilon_{0}\right]$, then if $\lambda^{\prime} \in G_{\epsilon, \gamma, \nu}^{(N)}(V)=[-1,1] \backslash B_{\epsilon, \gamma, \nu}^{(N)}(V)$, the following estimate holds

$$
\left\|\left(\Pi_{0} \Pi_{N} \mathcal{L}_{\epsilon, \lambda^{\prime}, \nu, V\left(\epsilon, \lambda^{\prime}, \nu\right)} \Pi_{N} \Pi_{0}\right)^{-1}\right\|_{0} \leq \frac{N^{\tau}}{\gamma} .
$$

Moreover, $B_{\epsilon, \gamma, \nu}^{(N)}(V)$ is the set of $\lambda^{\prime} \in[-1,1]$ such that $\left(\lambda^{\prime}, \nu\right) \in \Lambda$ and

$$
\left\|\left(\Pi_{0} \Pi_{N} \mathcal{L}_{\epsilon, \lambda^{\prime}, \nu, V\left(\epsilon, \lambda^{\prime}, \nu\right)} \Pi_{N} \Pi_{0}\right)^{-1}\right\|_{0}>\frac{N^{\tau}}{\gamma} .
$$

For a fixed $\nu$, such that $|\nu| \leq \epsilon_{0}$, let us define the "good set of $\lambda^{\prime \prime}$ for all $M_{\epsilon} \leq K \leq N$,

$$
\mathcal{G}_{\epsilon, \gamma, \nu}^{(N)}(V)=\cap_{M_{\epsilon}<K \leq N} G_{\epsilon, \gamma, \nu}^{(K)}(V)
$$

where $M_{\epsilon}$ is defined in (42), then we have the following

Lemma 21. Assume that $\gamma \leq \widetilde{\gamma}=1 /\left(2^{2 l_{0}+1} c_{0}\right)$ and $\nu$, such that $|\nu| \leq \epsilon_{0}$, and

$$
\tau-d-3-10 l_{0}>0 .
$$

Let $V \in \mathcal{U}_{M}^{(N)}$. Then $G_{\epsilon, \gamma, \nu}^{(K)}(V)=[-1,1]$ and $B_{\epsilon, \gamma, \nu}^{(K)}(V)=\emptyset$ if $K \leq M_{\epsilon}$. If $\lambda^{\prime} \in \mathcal{G}_{\epsilon, \gamma, \nu}^{(N)}(V)$ all eigenvalues of $\Pi_{0} \Pi_{K} \mathcal{L}_{\epsilon, \lambda^{\prime}, \nu, V\left(\epsilon, \lambda^{\prime}, \nu\right)} \Pi_{K} \Pi_{0}$ have absolute value $\geq \gamma K^{-\tau}$ for all $1 \leq K \leq N$. Moreover the measure of $\cup_{M_{\epsilon}<K \leq N} B_{\epsilon, \gamma, \nu}^{(K)}(V)$, i.e. the "bad set of $\lambda^{\prime}$ ", is bounded by

$$
c_{3}(\tau) \gamma \epsilon^{2} / M_{\epsilon}^{2} \text {. }
$$

Proof. If $N \geq 1$, then $2 c_{0} \gamma \leq \frac{1}{2^{2 l_{0}}} \leq \frac{N^{\tau}}{\left(1+N^{2}\right)^{2 l_{0}}}$, i.e.

$$
\frac{1}{2 c_{0}\left(1+N^{2}\right)^{2 l_{0}}} \geq \frac{\gamma}{N^{\tau}}
$$


The first assertions now follow for $N \leq M_{\epsilon}$ from (41). For $N>M_{\epsilon}$ the result follows from(56). The bad set satisfies

$$
\operatorname{meas}\left\{\cup_{M_{\epsilon}<K \leq N} B_{\epsilon, \gamma, \nu}^{(K)}(V)\right\} \leq \sum_{M_{\epsilon}<K \leq N} \frac{4 \gamma c_{1}}{\epsilon^{3} K^{\tau-d}} \leq \frac{4 \gamma c_{1}(\tau-d-1)}{\epsilon^{3} M_{\epsilon}^{\tau-d-1}},
$$

and from (42) we see that for $\tau-d-3-10 l_{0}>0$, the estimate of the bad set follows.

We need to obtain an estimate looking like (56), but in $\mathcal{H}_{s}$ for $s>0$, with an exponent on $N$ not depending on $s$. For getting such a good estimate, we need to show "separation properties" of "singular sites" which generate small divisors. This is the object of section 3 , which uses extensively the results of [BB10], [BBP10].

\section{Estimate of the inverse linearized operator in $\mathcal{H}_{s}$}

\subsection{Separation properties (H1) and (H2)}

The eigenvalues of the unperturbed operator $D_{N}=(1+\Delta)^{2} \mid E_{N}$ restricted to $E_{N}=\Pi_{N} \Pi_{0} \mathcal{H}_{0}$ are the numbers $\left(1-|\mathbf{k}|^{2}\right)^{2}$ where $|\mathbf{k}| \neq 1$, and $0 \leq N_{\mathbf{k}} \leq N$. Let $\rho>0$. We need to have good separation properties of the singular set

$$
S_{(N)}=\left\{\mathbf{k} \in \Gamma ;\left(1-|\mathbf{k}|^{2}\right)^{2}<\rho,|\mathbf{k}| \neq 1,0 \leq N_{\mathbf{k}} \leq N\right\},
$$

which contains the k's corresponding to the small denominators, whereas the regular set is

$$
R_{(N)}:=\left\{\mathbf{k} \in \Gamma ;\left(1-|\mathbf{k}|^{2}\right)^{2} \geq \rho,|\mathbf{k}| \neq 1,0 \leq N_{\mathbf{k}} \leq N\right\} .
$$

We have a bijection between $S_{(N)}$ and $S(N):=\left\{x \in \Gamma(N) ;\left(1-|\mathbf{k}(x)|^{2}\right)^{2}<\rho\right\}$ where $\mathbf{k}(x)$ is defined in (7) and

$$
\Gamma(N):=\left\{x \in \mathbb{Z}^{d} ; 0 \leq|x| \leq N, \mathbf{k}(x) \in \Gamma \backslash\left\{\mathbf{k}_{j}, j=1, \ldots, 2 q\right\}\right\} .
$$

Let $\mathbf{k}=\mathbf{k}(x), x=\left(m_{1}, . ., m_{d}\right) \in \mathbb{Z}^{d}$. Then $\mathfrak{d}^{2}|\mathbf{k}|^{2}=\sum_{s, t=1}^{d} m_{s} m_{t}\left\langle\mathbf{k}_{s}^{*}, \mathbf{k}_{t}^{*}\right\rangle$. Let $\theta_{0}:=\pi / q$, $\omega=2 \cos \theta_{0}$. Then $2\left\langle\mathbf{k}_{h}, \mathbf{k}_{j}\right\rangle=2 \cos \left\{(h-j) \theta_{0}\right\}$ and this is a polynomial with integer coefficients in $\omega$. Therefore $2\left\langle\mathbf{k}_{s}^{*}, \mathbf{k}_{t}^{*}\right\rangle$ and $\mathfrak{d}^{2}|\mathbf{k}|^{2}$ are polynomials in $\omega$ and the coefficients in the latter polynomial are integer-valued quadratic forms in $x$. Here $\omega$ is root of the minimal polynomial with integer coefficients of degree $d / 2=: l_{0}+1$ and leading coefficient 1 (cf.[IR10, (3),(4)]). By division through this minimal polynomial we see that there are integer-valued quadratic forms $P_{r}(x)$ such that

$$
\mathfrak{d}^{2}|\mathbf{k}(x)|^{2}=\sum_{r=0}^{l_{0}} P_{r}(x) \omega^{r}=: P(x) .
$$

Hence $P$ is bounded on $S(N)$. 
There exist symmetric matrices $A_{r}$ of $\mathcal{M}_{d}(\mathbb{Z})$ such that $P_{r}(x)=\left(x, A_{r} x\right)$ where $(.,$. denotes the usual scalar product of $\mathbb{Z}^{d}$ and with $A:=\sum_{r=0}^{l_{0}} A_{r} \omega^{r}$ we have $(x, A x)=$ $\mathfrak{d}^{2}|\mathbf{k}(\mathbf{x})|^{2}$. We note that $A$ is positive. So

$$
P(x)=\sum_{l=0}^{l_{0}}\left(x, A_{l} x\right) \omega^{l}=(x, A x), A_{l} \in \mathcal{M}_{d}(\mathbb{Z}), A=\sum_{l=0}^{l_{0}} A_{l} \omega^{l}>0 .
$$

Next we show that the singular set $S(N)$ can be partitioned into disjoint clusters satisfying Proposition 23 (see below). To this end we first adapt to our situation Bourgain's separation lemma [Bou95]. We follow its proof in W. Craig [Cra00, p. 110-113].

Lemma 22. Let $P$ be defined by (60) and $S$ be a subset of $\mathbb{Z}^{d}$ such that $P$ is bounded on $S$. Then there exists $r>0$ such that for all $B \geq 2$ and for any sequence $\left\{x_{j}\right\}_{j=0, \ldots, K}$ of distinct points of $S$ such that $\left|x_{j+1}-x_{j}\right|<B$, we have :

$$
K<B^{r} .
$$

Proof. It is sufficient to give the proof in the case that $K \geq K_{0}$ with $K_{0}$ sufficiently large. In the following $C$ denotes a positive constant which may be different in different formulas.

As in [Cra00, p.110] choose integers $J_{t}$ with $J_{0}=K>J_{1}>\ldots>J_{d}>1$ such that $J_{t+1}<J_{t} / 2$ and some other restriction which will be introduced lateron. This choice is possible if $K_{0}$ is chosen sufficiently large.

For every integer $j$ with $1 \leq j \leq K$ and every $t \in\{0,1, \ldots, d\}$ let $X(j, t)=\operatorname{span}\left\{\left(x_{l}-\right.\right.$ $\left.\left.x_{j}\right):|l-j| \leq J_{t}\right\}$. Let $d(t):=\min _{j} \operatorname{dim} X(j, t)$ be attained for $j=j(t)$. Then there exists $t_{0} \in\{0, \ldots, d-1\}$ such that $d\left(t_{0}\right)=d\left(t_{0}+1\right)$ and

$$
X\left(j, t_{0}+1\right)=X\left(j\left(t_{0}\right), t_{0}\right) \text { if }\left|j-j\left(t_{0}\right)\right|<J_{t_{0}+1} .
$$

For the proof see [Cra00, p. 110].

Let $j_{0}=j\left(t_{0}\right), X_{0}:=X\left(j_{0}, t_{0}\right), d_{0}=\operatorname{dim} X_{0}$. Since $X_{0}$ is spanned by $x_{l}-x_{j_{0}}$ with $\left|l-j_{0}\right|<J_{t_{0}+1}$ there is a basis $\left\{e_{l}\right\}_{l=1}^{d_{0}}$ for $X_{0}$ with $\left|e_{l}\right| \leq J_{t_{0}+1} B$.

Choose $j$ such that $\left|j-j_{0}\right| \leq J_{t_{0}}$ and $w=x_{j}-x_{j_{0}}$ has maximal length. Then there are $J_{t_{0}}$ distinct vectors $x_{h}-x_{j_{0}} \in X_{0} \cap \mathbb{Z}^{d}$ with length $\leq|w|$. From this it follows that

$$
J_{t_{0}} \leq C|w|^{d_{0}}
$$

with some constant $C$ depending only on $d$ (cf.[Cra00, (8.30)]). Next we derive an upper bound for $|w|$ in terms of $J_{t_{0}+1}$. We have $w=\sum_{l=1}^{d_{0}} a_{l} e_{l}$ for certain coefficients $a_{l}$. Then $\left\langle w, A e_{m}\right\rangle=\sum_{l=1}^{d_{0}} a_{l}\left\langle e_{l}, A e_{m}\right\rangle$ and from this we may solve for $a_{l}$ using Cramer's rule. Let $M$ be the matrix with elements $\left\langle e_{l}, A e_{m}\right\rangle$. Then $M$ is invertible since $A$ is positive. Let $M_{l}$ be the matrix obtained from $M$ by replacing the $l$-th column by the column of the $\left\langle w, A e_{m}\right\rangle$. Then $a_{l}=\operatorname{det} M_{l} / \operatorname{det} M$. From $\left\langle e_{l}, A e_{m}\right\rangle=\sum_{r=0}^{l_{0}} \omega^{r}\left\langle e_{l}, A_{r} e_{m}\right\rangle$ it follows that $\operatorname{det} M$ is a polynomial in $\omega$ of degree at most $d_{0} l_{0}$ with integer coefficients. These coefficients are bounded by $C_{0}\left(J_{t_{0}+1} B\right)^{2 d_{0}}$ since $\left|\left\langle e_{l}, A_{r} e_{m}\right\rangle\right| \leq C\left|e_{l}\right|\left|e_{m}\right| \leq C\left(J_{t_{0}+1} B\right)^{2}$. Using the minimal polynomial of $\omega$ we may show that $\operatorname{det} M$ may be written as a polynomial in $\omega$ of degree $l_{0}$ at most with similar estimates of the coefficients as above. Next we apply lemma 2.1 in [IR10] using the diophantine property of $\omega$ and obtain

$$
|\operatorname{det} M| \geq C\left(J_{t_{0}+1} B\right)^{-2 d_{0} l_{0}} .
$$


For an estimate of $M_{l}$ we use $\left|\left\langle e_{l}, A e_{m}\right\rangle\right| \leq C\left(J_{t_{0}+1} B\right)^{2}$ as above. We will prove lateron

$$
\left|\left\langle w, A e_{m}\right\rangle\right| \leq C\left(J_{t_{0}+1} B\right)^{2\left(d_{0}+1\right)} .
$$

From these estimates we deduce that $\left|\operatorname{det} M_{l}\right| \leq C\left(J_{t_{0}+1} B\right)^{4 d_{0}}$ and therefore $\left|a_{l}\right| \leq C^{\prime}\left(J_{t_{0}+1} B\right)^{\beta_{1}}$ where $\beta_{1}=2 d_{0}\left(l_{0}+2\right)$ and $|w| \leq C^{\prime}\left(J_{t_{0}+1} B\right)^{\beta_{1}+1}$, since $w=\sum_{l=1}^{d_{0}} a_{l} e_{l}$. Combining this with (63) we obtain

$$
J_{t_{0}} \leq C\left(J_{t_{0}+1} B\right)^{\beta}
$$

where $\beta=d_{0}\left(\beta_{1}+1\right)$. Next we use the freedom in the choice of the numbers $J_{t}$ and choose these in such a way that for some $r_{1}>0$ one has $K^{r_{1}}<C J_{t} J_{t+1}^{-\beta}$ as in [Cra00, p. 113]. This is possible for $K \geq K_{0}$ with $K_{0}$ sufficiently large. From this and (65) we obtain the estimate of the lemma.

For the proof of (64) we use the projection $\Pi_{X_{0}}$ of $\mathbb{Z}^{d}$ onto $X_{0}$ and estimate first $\Pi_{X_{0}}(A w)$. Since $\Pi_{X_{0}}\left(A x_{j_{0}}\right)=\sum_{l=1}^{d_{0}} b_{l} e_{l}$ for certain $b_{l}$ we have $\left\langle A x_{j_{0}}, e_{m}\right\rangle=\sum_{l=1}^{d_{0}} b_{l}\left\langle e_{l}, e_{m}\right\rangle$. Here the lefthand side equals $\left\langle A x_{j_{0}}, x_{h}-x_{j_{0}}\right\rangle$ for some $h=h(m)$ with $\left|h-j_{0}\right| \leq J_{t_{0}+1}$. Next we use $2\left\langle A x_{l}, x_{h}-x_{l}\right\rangle=P\left(x_{h}\right)-P\left(x_{l}\right)-P\left(x_{h}-x_{l}\right)$ where $\left|P\left(x_{h}-x_{l}\right)\right| \leq C\left|x_{h}-x_{l}\right|^{2} \leq$ $C(|h-l| B)^{2}$ and $P(x)$ is bounded on $S$. Since $|h-l| \geq 1$ when $h \neq l$, we have indeed $\left|\left\langle A x_{l}, x_{h}-x_{l}\right\rangle\right| \leq C(|h-l| B)^{2}$, and in particular $\left|\left\langle A x_{j_{0}}, e_{m}\right\rangle\right| \leq C\left(J_{t_{0}+1} B\right)^{2}$. Furthermore, $\operatorname{det}\left(\left\langle e_{l}, e_{m}\right\rangle\right) \neq 0$ and the $\left\langle e_{l}, e_{m}\right\rangle$ are integers, so the determinant has absolute value $\geq 1$. Again using Cramer's rule with the estimates above we see that $\left|b_{l}\right| \leq C\left(J_{t_{0}+1} B\right)^{2 d_{0}}$ and therefore $\left|\Pi_{X_{0}}\left(A x_{j_{0}}\right)\right| \leq C\left(J_{t_{0}+1} B\right)^{2 d_{0}+1}$. Similarly one may show the same estimate with $j_{0}$ replaced by $j$ using a different basis for $X_{0}$. Hence $\left|\Pi_{X_{0}}(A w)\right| \leq C\left(J_{t_{0}+1} B\right)^{2 d_{0}+1}$ and so $\left\langle A w, e_{m}\right\rangle=\left\langle w, A e_{m}\right\rangle$ satisfies (64).

From this lemma we may derive as in [BB10]

Proposition 23. There exists $\rho_{0}>0$ independent of $N$ such that if $\left.\left.\rho \in\right] 0, \rho_{0}\right]$ then there exists a decomposition of $S(N)=\bigcup_{\alpha \in \mathcal{A}} \Omega_{\alpha}$ into a union of disjoint clusters $\Omega_{\alpha}$ satisfying:

- (H1), for all $\alpha \in \mathcal{A}, M_{\alpha} \leq 2 m_{\alpha}$ where $M_{\alpha}=\max _{x \in \Omega_{\alpha}}|x|$ and $m_{\alpha}=\min _{x \in \Omega_{\alpha}}|x|$;

- $(H 2)$, there exists $\delta=\delta(d) \in] 0,1[$ independent of $N$ such that if $\alpha, \beta \in \mathcal{A}, \alpha \neq \beta$ then

$$
\operatorname{dist}\left(\Omega_{\alpha}, \Omega_{\beta}\right):=\min _{x \in \Omega_{\alpha}, y \in \Omega_{\beta}}|x-y| \geq \frac{\left(M_{\alpha}+M_{\beta}\right)^{\delta}}{2}
$$

Proof. The proof is an adaptation of that of lemma 4.4 of [BB10] as follows: Since $P$ is bounded on $S(N)$ we may apply lemma 22 with $S=S(N)$. Let $\delta=\{2(r+1)\}^{-1}$ where $r$ is the number given by that lemma. Then two elements $x$ and $y$ in $S(N)$ are said to be equivalent if there exist $x_{l} \in S(N), l=0,1, \ldots, n$ with $x_{0}=x, x_{n}=y$ and $\left|x_{l+1}-x_{l}\right| \leq\left(\left|x_{l}\right|+\left|x_{l+1}\right|\right)^{\delta}, \forall l$. Thus we get a decomposition of $S(N)$ in disjoint equivalence classes $\Omega_{\alpha}$. Consider $z_{\alpha} \in \Omega_{\alpha}$ such that $\left|z_{\alpha}\right|=\max _{z \in \Omega_{\alpha}}|z|=: M_{\alpha}$. Each $x \in \Omega_{\alpha}$ is connected to $z_{\alpha}$ by a $B$-chain with $B:=\left(2 M_{\alpha}\right)^{\delta}$ as in lemma 22 . This lemma implies that if $K$ is the number of elements in this chain then $K<B^{r}$ and therefore

$$
|x| \geq\left|z_{\alpha}\right|-K B>M_{\alpha}-B^{r+1}=M_{\alpha}-\left(2 M_{\alpha}\right)^{\delta(r+1)}=M_{\alpha}\left(1-\left(2 / M_{\alpha}\right)^{1 / 2}\right),
$$


since $\delta(r+1)=1 / 2$. Hence if $M_{\alpha} \geq 8$ then $|x| \geq M_{\alpha} / 2$ and in particular $m_{\alpha} \geq M_{\alpha} / 2$. We next choose $\rho$ such that for all $x \in S(N)$ we have $|x| \geq 8$ and then the proof is complete.

From (12) and the definition of $N_{\mathbf{k}}$ we have for $x \in S(N)$

$$
\rho>\left(1-|\mathbf{k}(x)|^{2}\right)^{2} \geq \frac{c^{2}}{\left(1+|x|^{2}\right)^{2 l_{0}}},
$$

hence for $\left(\frac{c^{2}}{\rho}\right)^{1 / 2 l_{0}} \geq 65$, which holds for $\rho<\rho_{0}$ small enough, we have $|x|>8$, and therefore $M_{\alpha} \geq 8$.

The proof of $(H 2)$ is immediate: for any $x \in \Omega_{\alpha}, y \in \Omega_{\beta}$, we have

$$
|x-y|>(|x|+|y|)^{\delta} \geq\left(m_{\alpha}+m_{\beta}\right)^{\delta}>(1 / 2)\left(M_{\alpha}+M_{\beta}\right)^{\delta} .
$$

\subsection{Estimate in $\Pi_{N} \Pi_{0} \mathcal{H}_{s}$ of $\left(\Pi_{0} \Pi_{N} \mathcal{L}_{\epsilon, \lambda^{\prime}, \nu, V} \Pi_{N} \Pi_{0}\right)^{-1}$}

We can prove the following

Proposition 24. Let $d=2\left(l_{0}+1\right)$ be the dimension of the $\mathbb{Q}$ - vector space spanned by the wave vectors $\mathbf{k}_{j}, j=1, \ldots, 2 q$, and let $\tau>d+3+10 l_{0}=12 l_{0}+5$ as in Lemma 21 . Let $s_{0} \geq \frac{d}{2}+\frac{d+\tau}{\delta}+1$, where $\delta$ is the number introduced in separation property (H2), and define $\mu:=2 \tau+3 d / 2$. Assume moreover that $0<\gamma \leq \widetilde{\gamma}=1 /\left(2^{2 l_{0}+1} c_{0}\right)$, and $\left(\epsilon, \lambda^{\prime}, \nu, V\right) \in$ $\left[0, \epsilon_{1}\right] \times \Lambda \times \mathcal{U}_{M}^{(N)}$, with $\lambda^{\prime} \in \mathcal{G}_{\epsilon, \gamma, \nu}^{(N)}(V)$ as defined by (57), $\epsilon_{1}$ small enough. Let $\bar{s}>s_{0}$. Then for all $s \in\left[s_{0}, \bar{s}\right]$ there exists $K(s)>0$ such that for any $h \in \Pi_{N} \Pi_{0} \mathcal{H}_{s}$, we have

$$
\left\|\left(\Pi_{0} \Pi_{N} \mathcal{L}_{\epsilon, \lambda^{\prime}, \nu, V\left(\epsilon, \lambda^{\prime}, \nu\right)} \Pi_{N} \Pi_{0}\right)^{-1} h\right\|_{s} \leq K(s) \frac{N^{\mu}}{\gamma}\left(\|h\|_{s}+\left\|V\left(\epsilon, \lambda^{\prime}, \nu\right)\right\|\left\|_{s}\right\| h \|_{s_{0}}\right) .
$$

Proof. Lemma 21 applies here and guarantees that for any $1 \leq K \leq N$ all eigenvalues of $\Pi_{0} \Pi_{K} \mathcal{L}_{\epsilon, \lambda^{\prime}, \nu, V} \Pi_{K} \Pi_{0}$ are $\geq \gamma K^{-\tau}$.

Then we notice that the scale of Sobolev spaces $\Pi_{0} \mathcal{H}_{s}$ satisfies

- $\forall s \leq s^{\prime}, \Pi_{0} \mathcal{H}_{s^{\prime}} \subseteq \Pi_{0} \mathcal{H}_{s}$,

- $\forall V \in \Pi_{0} \mathcal{H}_{s^{\prime}}, \quad\|V\|_{s} \leq\|V\|_{s^{\prime}}$,

- $E_{N}=\Pi_{N} \Pi_{0} \mathcal{H}_{0}, N \geq 0$ is a closed subspace of $\Pi_{0} \mathcal{H}_{s}, \cup_{N \geq 0} E_{N}$ dense in $\Pi_{0} \mathcal{H}_{s}$ for $s \geq$ 0 .

There exists a constant $C(s, d)>0$, such that the projection operator $\Pi_{N}$ plays the role of a smoothing operator:

$$
\begin{aligned}
& \forall V \in \Pi_{0} \mathcal{H}_{s},\left\|\Pi_{N} V\right\|_{s+d} \leq C(s, d) N^{d}\|V\|_{s}, \\
& \forall V \in \Pi_{0} \mathcal{H}_{s+d},\left\|\left(\mathbb{I}-\Pi_{N}\right) V\right\|_{s} \leq C(s, d) N^{-d}\|V\|_{s+d}
\end{aligned}
$$

Moreover we satisfy separation properties (H1) and (H2) for the singular sites (see previous subsection), and the non diagonal part of operator $\Pi_{0} \Pi_{N} \mathcal{L}_{\epsilon, \lambda^{\prime}, \nu, V} \Pi_{N} \Pi_{0}$ is $O\left(\epsilon^{2}\right)$ since it is included into the operator $\epsilon^{2} T\left(\epsilon, \lambda^{\prime}, \nu, V\right)$. Moreover the operators satisfy good "tame properties" (see subsection 2.5), so that we may apply directly Proposition 3.1 p.625 of [BB10] just replacing the dimension $d+1$ in this paper, by $d=2\left(l_{0}+1\right)$ and using extra parameters. 


\section{Resolution of the range equation}

In this section we use [BBP10] for finding a solution $V\left(\epsilon, \lambda^{\prime}, \nu\right)$ bounded by $O(\epsilon)$, of class $C^{1}$ in its arguments, defined for $\left(\epsilon, \lambda^{\prime}, \nu\right)$ in a suitably large subset of $\left(0, \epsilon_{1}\right) \times \Lambda$.

Indeed, we have (see subsections 2.5 and 3.2) the good functional setting and the good "tame" properties of the map $\left(\epsilon, \lambda^{\prime}, \nu, V\right) \mapsto \mathcal{F}\left(\epsilon, \lambda^{\prime}, \nu, V\right)$ from $\left(0, \epsilon_{1}\right) \times \Lambda \times \Pi_{0} \mathcal{H}_{s+4}$ into $\Pi_{0} \mathcal{H}_{s}$ for $s \geq s_{0}$. Moreover, for any $\left(\lambda^{\prime}, \nu\right) \in \Lambda$ we have

$$
\mathcal{F}\left(0, \lambda^{\prime}, \nu, 0\right)=0 .
$$

Then we prove the following

Proposition 25. Choose $N_{2} \geq N_{1} \geq M_{\epsilon}$, and $V_{1} \in \mathcal{U}_{M}^{\left(N_{1}\right)}, V_{2} \in \mathcal{U}_{M}^{\left(N_{2}\right)}$ and for $(\epsilon, \nu) \in$ $\left(0, \epsilon_{1}\right) \times\left[-\epsilon_{0}, \epsilon_{0}\right]$ consider the set of $\lambda^{\prime}$ which are "good" for $V_{1}$ but "bad" for $V_{2}:\left(\mathcal{G}_{\epsilon, \gamma, \nu}^{\left(N_{2}\right)}\left(V_{2}\right)\right)^{c} \cap$ $\mathcal{G}_{\epsilon, \gamma, \nu}^{\left(N_{1}\right)}\left(V_{1}\right)$. Assume that $\left\|V_{2}-V_{1}\right\|_{s_{0}} \leq N_{1}^{-\sigma}$, with $\sigma>d+3$, and $\tau>d+3+10 l_{0}$, then for $\epsilon_{1}$ small enough and in particular $\epsilon_{1} \leq \gamma^{2 l_{0}}$ :

$$
\left.\operatorname{meas}\left(\left(\mathcal{G}_{\epsilon, \gamma, \nu}^{\left(N_{2}\right)}\left(V_{2}\right)\right)^{c} \cap \mathcal{G}_{\epsilon, \gamma, \nu}^{\left(N_{1}\right)}\left(V_{1}\right)\right) \cap[-1,1]\right) \leq C_{1} \frac{\gamma \epsilon^{2}}{N_{1}},
$$

where the apex $c$ denotes the complementary in $[-1,1]$.

Proof. We have by construction

$$
\begin{aligned}
\left(\mathcal{G}_{\epsilon, \gamma, \nu}^{\left(N_{2}\right)}\left(V_{2}\right)\right)^{c} \cap \mathcal{G}_{\epsilon, \gamma, \nu}^{\left(N_{1}\right)}\left(V_{1}\right) & =\left(\cup_{M_{\epsilon} \leq K \leq N_{2}} B_{\epsilon, \gamma, \nu}^{(K)}\left(V_{2}\right)\right) \cap\left(\cap_{M_{\epsilon} \leq K \leq N_{1}} G_{\epsilon, \gamma, \nu}^{(K)}\left(V_{1}\right)\right) \\
& \subset\left(\cup_{M_{\epsilon} \leq K \leq N_{1}}\left(B_{\epsilon, \gamma, \nu}^{(K)}\left(V_{2}\right) \cap G_{\epsilon, \gamma, \nu}^{(K)}\right)\left(V_{1}\right)\right) \cup\left(\cup_{N_{1}<K \leq N_{2}} B_{\epsilon, \gamma, \nu}^{(K)}\left(V_{2}\right)\right) .
\end{aligned}
$$

Moreover, according to (36) there exists a constant $c>0$, such that for $\epsilon_{0}$ small enough and $K \leq N_{1}$ :

$$
\begin{aligned}
\left\|\Pi_{K} \Pi_{0} \mathcal{L}_{\epsilon, \lambda^{\prime}, \nu, V_{2}\left(\epsilon, \lambda^{\prime}, \nu\right)} \Pi_{K} \Pi_{0}-\Pi_{K} \Pi_{0} \mathcal{L}_{\epsilon, \lambda^{\prime}, \nu, V_{1}\left(\epsilon, \lambda^{\prime}, \nu\right)} \Pi_{K} \Pi_{0}\right\|_{0} & \leq c \epsilon^{5}\left\|V_{2}-V_{1}\right\|_{s_{0}} \\
& \leq \frac{c \epsilon^{5}}{N_{1}^{\sigma}}
\end{aligned}
$$

Let us assume that $\lambda^{\prime} \in B_{\epsilon, \gamma, \nu}^{(K)}\left(V_{2}\right) \cap G_{\epsilon, \gamma, \nu}^{(K)}\left(V_{1}\right)$, then there is at least one eigenvalue of $\Pi_{K} \Pi_{0} \mathcal{L}_{\epsilon, \lambda^{\prime}, \nu, V_{2}\left(\epsilon, \lambda^{\prime}, \nu\right)} \Pi_{K} \Pi_{0}$ of modulus $<\frac{\gamma}{K^{\tau}}$. According to the perturbation theory, [Kato] p.61 theorem 6.44, $\Pi_{K} \Pi_{0} \mathcal{L}_{\epsilon, \lambda^{\prime}, \nu, V_{1}\left(\epsilon, \lambda^{\prime}, \nu\right)} \Pi_{K} \Pi_{0}$ has an eigenvalue of modulus less than $\frac{\gamma}{K^{\tau}}+$ $\frac{c \epsilon^{5}}{N_{1}^{\sigma}}$. Since $\lambda^{\prime} \in G_{\epsilon, \gamma, \nu}^{(K)}\left(V_{1}\right)$ then this eigenvalue is in fact of modulus lying in the interval

$$
\left[\frac{\gamma}{K^{\tau}}, \frac{\gamma}{K^{\tau}}+\frac{c \epsilon^{5}}{N_{1}^{\sigma}}\right)
$$

We need now to give a bound for the measure of the ('bad") set of $\lambda^{\prime}$ corresponding to such an interval for eigenvalues of $\Pi_{K} \Pi_{0} \mathcal{L}_{\epsilon, \lambda^{\prime}, \nu, V_{1}\left(\epsilon, \lambda^{\prime}, \nu\right)} \Pi_{K} \Pi_{0}$. Indeed, according to (45)

$$
\partial_{\lambda^{\prime}} \Pi_{K} \Pi_{0} \mathcal{L}_{\epsilon, \lambda^{\prime}, \nu, V_{1}\left(\epsilon, \lambda^{\prime}, \nu\right)} \Pi_{K} \Pi_{0}-\epsilon^{3} \mathbb{I}=\mathcal{O}\left(\epsilon^{5}\right)
$$


Hence, the Lipschitz constant of any eigenvalue of $\Pi_{K} \Pi_{0} \mathcal{L}_{\epsilon, \lambda^{\prime}, \nu, V_{1}\left(\epsilon, \lambda^{\prime}, \nu\right)} \Pi_{K} \Pi_{0}$ with respect to $\lambda^{\prime}$ is bounded from below by $\epsilon^{3} / 2$. This means that this "bad" set of $\lambda^{\prime}$ has a measure bounded by $\frac{2 c \epsilon^{2}}{N_{1}^{\sigma}}$. In summing for all eigenvalues, and for $M_{\epsilon} \leq K \leq N_{1}$ it results that

$$
\text { meas } \cup_{M_{\epsilon} \leq K \leq N_{1}}\left(B_{\epsilon, \gamma, \nu}^{(K)}\left(V_{2}\right) \cap G_{\epsilon, \gamma, \nu}^{(K)}\right)\left(V_{1}\right) \leq \frac{2 c \epsilon^{2}}{N_{1}^{\sigma}} \sum_{M_{\epsilon} \leq K \leq N_{1}} c_{1} K^{d} \leq \frac{2 c c_{1} \epsilon^{2}}{N_{1}^{\sigma-d-1}} \text {. }
$$

Since $\epsilon \leq \gamma^{2 l_{0}}$, we have for $c_{2}>0$

$$
1 / N_{1} \leq 1 / M_{\epsilon} \leq c_{2} \gamma
$$

hence, since $\sigma \geq d+3$, we have $N_{1}^{\sigma-d-1} \geq N_{1} /\left(c_{2} \gamma\right)$. Now, we also have (see (54))

$$
\text { meas } \cup_{N_{1}<K \leq N_{2}} B_{\epsilon, \gamma, \nu}^{(K)}\left(V_{2}\right) \leq \sum_{N_{1}<K \leq N_{2}} \frac{4 \gamma c_{1}}{\epsilon^{3} K^{\tau-d}} \leq \frac{4 \gamma c_{1}(\tau-d-1)}{\epsilon^{3} N_{1}^{\tau-d-1}},
$$

hence, since $\tau>d+3+10 l_{0}$, we have for $N_{1} \geq M_{\epsilon}$

$$
\epsilon^{3} N_{1}^{\tau-d-1} \geq \epsilon^{3} M_{\epsilon}^{10 l_{0}} N_{1}^{\tau-d-1-10 l_{0}} \geq \widetilde{c}_{2} N_{1}^{2} / \epsilon^{2}
$$

and there exists a constant $C_{1}$ such that we have

$$
\frac{2 c c_{1} \epsilon^{2}}{N_{1}^{\sigma-d-1}}+\frac{4 \gamma c_{1}(\tau-d-1)}{\epsilon^{3} N_{1}^{\tau-d-1}} \leq C_{1} \gamma \epsilon^{2} / N_{1}
$$

Proposition 25 is proved.

We may apply theorem 3 of Berti-Bolle-Procesi ([BBP10]) to equation $\mathcal{F}\left(\epsilon, \lambda^{\prime}, \nu, V\right)=0$. Let $\gamma, \tau, \mu, s_{0}$ be as in Proposition 24. Moreover, let $\bar{s}:=s_{0}+4(\mu+5)+2 \sigma, \sigma>4(\mu+4)$.

From proposition 24 , it follows that if $\left(\epsilon, \lambda^{\prime}, \nu, V\right) \in\left[0, \epsilon_{1}\right] \times \Lambda \times \mathcal{U}_{M}^{(N)}$ and $\lambda^{\prime} \in \mathcal{G}_{\epsilon, \gamma, \nu}^{(N)}(V)$ then $\left(\epsilon, \lambda^{\prime}, \nu, V\left(\epsilon, \lambda^{\prime}, \nu\right)\right) \in J_{N, \gamma, \mu, \mathcal{K}}$ (as defined in (4) of [BBP10], that is (66) holds for $s \in\left[s_{0}, \bar{s}\right]$.

In [BBP] [theorem 3] one considers $N \geq N_{0}=N_{0}(\gamma)$ with $N_{0}(\gamma)$ sufficiently large and $0<\epsilon \leq \epsilon_{2}(\gamma)$ with $\epsilon_{2}(\gamma)$ sufficiently small. We may choose $N_{0}=N_{0}(\gamma)=M_{\epsilon_{3}(\gamma)}$ with a suitable $\epsilon_{3}(\gamma) \leq \epsilon_{2}$ and we consider in the following $0<\epsilon \leq \epsilon_{3}(\gamma)$.

Theorem 26. Let $s_{0}$ and $\tilde{\gamma}$ be as in Proposition 24. Then for all $0<\gamma<\tilde{\gamma}$ there exist $\epsilon_{3}(\gamma) \in\left[0, \epsilon_{0}\right]$ and a $C^{1}-\operatorname{map} V:\left(0, \epsilon_{3}(\gamma)\right) \times \Lambda \rightarrow \mathcal{H}_{s_{0}+4}$ such that $V\left(0, \lambda^{\prime}, \nu\right)=0$ and if $\epsilon \in\left(0, \epsilon_{3}(\gamma)\right),\left(\lambda^{\prime}, \nu\right) \in \Lambda, \lambda^{\prime} \in\left([-1,1] \backslash C_{\epsilon, \gamma, \nu}\right)$, the function $V\left(\epsilon, \lambda^{\prime}, \nu\right)$ is solution of (32). Here $C_{\epsilon, \gamma, \nu}$ is a subset of $[-1,1]$ which is a Lipschitz function of $\nu$, and has Lebesgue-measure less than $C \gamma \epsilon^{2}$ for some constant $C>0$ independent of $\epsilon, \nu$ and $\gamma$.

Proof. In [BBP10] [theorem 3] the authors construct a sequence $\left\{V_{n}\right\}_{n \geq 0}$ of $C^{1}$ functions that converges uniformly in $C^{1}\left(\left[0, \epsilon_{3}(\gamma)\right) \times \Lambda\right)$ to a $C^{1}$ function $V$. When restricted to a set $A_{\infty}$ of parameters, $V\left(\epsilon, \lambda^{\prime}, \nu\right)$ satisfies $\mathcal{F}\left(\epsilon, \lambda^{\prime}, \nu, V\left(\epsilon, \lambda^{\prime}, \nu\right)\right)=0$.

We now use some more details of the cited theorem 3 of Berti, Bolle and Procesi. They define $N_{n}=N_{0}^{2^{n}}$ and $E_{(n)}=E_{N_{n}}$. Then $V_{n}\left(\epsilon, \lambda^{\prime}, \nu\right) \in E_{(n)}$ and $\left\|V_{n}-V_{n-1}\right\|_{s_{0}} \leq N_{n}^{-\sigma-1}$ 
for $n \geq 1$ where $\sigma>d+3$ as in Proposition 25. Let $\mathbf{G}_{\gamma}^{\left(N_{n}\right)}\left(V_{n-1}\right)=\left\{\left(\epsilon, \lambda^{\prime}, \nu\right) ; 0<\right.$ $\left.\epsilon<\epsilon_{3}(\gamma), \lambda^{\prime} \in \mathcal{G}_{\epsilon, \gamma, \nu}^{\left(N_{n}\right)}\left(V_{n-1}\right),|\nu| \leq \epsilon_{0}\right\}$ where $V_{-1}=0$. Then Proposition 24 implies that $\mathbf{G}_{\gamma}^{\left(N_{n}\right)}\left(V_{n-1}\right)$ satisfies the properties of $J_{\gamma, \mu, \mathcal{K}}^{(N)}$ as defined in (10) on p.382 of [BBP10]. In that paper the authors define $A_{\infty}:=\cap_{k=0}^{\infty} \mathbf{G}_{\gamma}^{\left(N_{k}\right)}\left(V_{k-1}\right)$.

By Lemma 21 we have $\mathcal{G}_{\epsilon, \gamma, \nu}^{\left(N_{0}\right)}(0)=[-1,1]$ and therefore $\mathbf{G}_{\gamma}^{\left(N_{0}\right)}(0)=\left(0, \epsilon_{3}\right) \times \Lambda$. From Proposition 25 we have

$$
\operatorname{meas}\left(\left(\mathcal{G}_{\epsilon, \gamma, \nu}^{\left(N_{n+1}\right)}\left(V_{n}\right)\right)^{c} \cap \mathcal{G}_{\epsilon, \gamma, \nu}^{\left(N_{n}\right)}\left(V_{n-1}\right)\right) \leq C_{1} N_{n}^{-1} \gamma \epsilon^{2} .
$$

We use an analogue of section 2.5 of [BBP10], to derive properties of $A_{\infty}$. Let us set $G_{n, \epsilon, \gamma, \nu}:=\mathcal{G}_{\epsilon, \gamma, \nu}^{\left(N_{n}\right)}\left(V_{n-1}\right)$. Let $C_{\epsilon, \gamma, \nu}=\left(\cap_{n=0}^{\infty} G_{n, \epsilon, \gamma, \nu}\right)^{c}$. Then $A_{\infty}=\left\{\left(\epsilon, \lambda^{\prime}, \nu\right) ; 0<\epsilon<\right.$ $\left.\epsilon_{3}(\gamma),\left(\lambda^{\prime}, \nu\right) \in \Lambda, \lambda^{\prime} \in\left([-1,1] \backslash C_{\epsilon, \gamma, \nu}\right)\right\}$ and we estimate the measure of $C_{\epsilon, \gamma, \nu}$. For this we use

$$
C_{\epsilon, \gamma, \nu} \subset \cup_{n \geq 1}\left(G_{n, \epsilon, \gamma, \nu}\right)^{c} \subset \cup_{n \geq 1}\left(\left(G_{n, \epsilon, \gamma, \nu}\right)^{c} \backslash\left(G_{n-1, \epsilon, \gamma, \nu}\right)^{c}\right) .
$$

Hence by (67) this set has measure at most

$$
C_{1} \gamma \epsilon^{2} \sum_{n \geq 0} \frac{1}{N_{n}} \leq C \gamma \epsilon^{2}
$$

which completes the proof of the theorem.

\section{Resolution of the bifurcation equation and proof of theo- rem 1}

Let $V$ be the function obtained in theorem 26. It is $C^{1}$ in all the parameters $\left(\epsilon, \lambda^{\prime}, \nu\right)$. Replacing $V\left(\epsilon, \lambda^{\prime}, \nu\right)$ in the bifurcation equation (31), we can solve with respect to $\lambda^{\prime}$ and find a function $h(\epsilon, \nu)$ which is $C^{1}$ in $(\epsilon, \nu)$, such that

$$
\lambda^{\prime}(\epsilon, \nu)=-2 \nu \lambda_{2}-\epsilon \lambda_{4}+\epsilon^{2} h(\epsilon, \nu),
$$

for $(\epsilon, \nu) \in\left(0, \epsilon_{3}(\gamma)\right) \times\left[-\epsilon_{0}, \epsilon_{0}\right]$ provided that $\epsilon_{3}$ is small enough, and $\left(\lambda^{\prime}, \nu\right) \in \Lambda$.

For a fixed $\epsilon$, when $\nu$ describes the part of the interval $\left[-\epsilon_{0}, \epsilon_{0}\right]$ such that $\left(\lambda^{\prime}, \nu\right) \in \Lambda$, $\lambda^{\prime}(\epsilon, \nu)$ describes a curve looking like a line, in the sector $\Lambda$ of the plane $\left(\nu, \lambda^{\prime}\right)$. Such a curve connects the two end points on $\nu= \pm \epsilon_{0} \lambda^{\prime}$ (recall that $\lambda_{4}<0$ )

$$
\begin{aligned}
& \left(-\epsilon_{0}\left(\frac{-\epsilon \lambda_{4}}{1-2 \epsilon_{0} \lambda_{2}}+\mathcal{O}^{-}\left(\epsilon^{2}\right)\right), \frac{-\epsilon \lambda_{4}}{1-2 \epsilon_{0} \lambda_{2}}+\mathcal{O}^{-}\left(\epsilon^{2}\right)\right) \\
& \left(\epsilon_{0}\left(\frac{-\epsilon \lambda_{4}}{1+2 \epsilon_{0} \lambda_{2}}+\mathcal{O}^{+}\left(\epsilon^{2}\right)\right), \frac{-\epsilon \lambda_{4}}{1+2 \epsilon_{0} \lambda_{2}}+\mathcal{O}^{+}\left(\epsilon^{2}\right)\right) .
\end{aligned}
$$

The values of $\lambda^{\prime}$ vary over an interval of length of order $\mathcal{O}\left(\epsilon \epsilon_{0}\right)$. According to theorem 26 , the measure of the bad set of $\lambda^{\prime}$ is bounded for any fixed $\nu$ as meas $C_{\epsilon, \gamma, \nu} \leq C \gamma \epsilon^{2}$. Moreover, we know that, in the plane $\left(\nu, \lambda^{\prime}\right)$ the bad set is located into "bad strips" $B S:=$ 
$\cup_{n \geq 1} B S_{N_{n}}\left(V_{n-1}\right)$ as defined in (53), for which we control the slopes (close to $-\alpha_{j}^{(N)}$ ). We are lead to use Condition 2. Indeed, as we shall see in Remark 28, Condition 2 implies that

$$
\left|\alpha_{j}^{(N)}\left(\epsilon, \lambda^{\prime}, \nu\right)-2 \lambda_{2}\right| \geq 6 c_{1}^{\prime},
$$

where $c_{1}^{\prime}$ is $\epsilon$ - close to $c_{1}$.

This assumption guarantees the transversality between the "line" (68) and the nearly straight bad strips $B S$. Indeed denoting by $\theta$ the angle of intersection of a bad strip with the curve (68) in the plane $\left(\nu, \lambda^{\prime}\right)$, we have $\tan \theta>c_{2}>0$ (independently of $N$ ). This comes from $\theta=\theta_{1}-\theta_{2}, \tan \left(\theta_{1}\right) \sim-2 \lambda_{2} \neq 0$ and $\tan \left(\theta_{2}\right) \sim-\alpha_{j}^{(N)}\left(\epsilon, \lambda^{\prime}, \nu\right)$, and

$$
\tan \theta=\frac{\tan \left(\theta_{1}\right)-\tan \left(\theta_{2}\right)}{1+\tan \left(\theta_{1}\right) \tan \left(\theta_{2}\right)}
$$

where the only possible problem occurs when $\theta_{1} \sim \theta_{2}$.

Each intersection of a bad strip $B S_{N_{n}}\left(V_{n-1}\right)$ of width $\delta \leq \frac{4 \gamma}{\epsilon^{3} N_{n}^{\tau}}$ with the line (68) gives only one segment of length bounded by $c \delta$, where $c$ is a constant independent of $N$ (a bound is obtained in dividing the thickness of the strip by the sine of $\theta$ ). Summing over all bad strips (for all $j$ and $N_{n}$ ), as this was done at Proposition 25 and Theorem 26, we obtain a union of bad segments on the line (68) and the measure of this set can be estimated, which gives a total measure of the bad segments, for $\nu$ fixed, bounded by $C \gamma \epsilon^{2}$. Projecting this set on the $\lambda^{\prime}$ axis gives the bad set for $\lambda^{\prime}$, centered at $-\epsilon \lambda_{4}$. The measure of this bad set is smaller than $C \gamma \epsilon^{2}$. The complement of this bad set is the good set for $\lambda^{\prime}$.

Then we get a set of good $\lambda^{\prime}$ with large measure, and corresponding good $\nu$ (following (68)) (see Figure 2).

Now we observe that we can write $\lambda^{\prime}=\epsilon \bar{\lambda}$, with $\bar{\lambda}$ centered in $-\lambda_{4}$ and $\nu=\epsilon \bar{\nu}$ with $|\bar{\nu}| \leq \epsilon_{0}$. This defines the good set $\bar{\Lambda}_{\epsilon}$ of all good $\bar{\lambda}_{\epsilon}$, for any fixed $\epsilon$, and correspondingly the good $\bar{\nu}(\epsilon)$. Moreover, as $\bar{\lambda}$ varies now on an interval of length of order $\mathcal{O}\left(\epsilon_{0}\right)$, the measure of the bad set of $\bar{\lambda}$ is now bounded by $C^{\prime} \gamma \epsilon$. We recall that $\epsilon<\epsilon_{3}(\gamma)$ which tends towards 0 as $\gamma$ tends towards 0 , and we notice that $C^{\prime} \gamma \epsilon / \epsilon_{0}<C^{\prime} \gamma$ as soon as $\epsilon \leq \epsilon_{0}$. It results that we have

$$
\frac{1}{2 \epsilon_{0}} \text { meas }\left\{\bar{\Lambda}_{\epsilon}^{c} \cap\left(-\lambda_{4}-\epsilon_{0},-\lambda_{4}+\epsilon_{0}\right)\right\} \leq C^{\prime} \gamma,
$$

and, since $\gamma$ is independent of $\epsilon_{0}$,

$$
\frac{1}{2 \epsilon} \operatorname{meas}\left\{\bar{\Lambda}_{\epsilon} \cap\left(-\lambda_{4}-\epsilon,-\lambda_{4}+\epsilon\right)\right\} \underset{\epsilon \rightarrow 0}{\rightarrow} 1 .
$$

Then the existence of a solution $\left(\epsilon, \lambda^{\prime}(\epsilon, \nu(\epsilon)), \nu(\epsilon)\right)$ of (68), persists as $\epsilon$ tends towards 0 , even though we don't know anything about its continuity in $\epsilon$.

Finally, due to (21) and (28), we obtain a solution of (2) under the form

$$
\begin{aligned}
U & =\epsilon u_{0}+\epsilon^{3} u_{1}+\epsilon^{3} \bar{\nu}(\epsilon)\left[u_{0}+3 \epsilon^{2} u_{1}\right]+\epsilon^{4} V(\epsilon, \epsilon \bar{\lambda}(\epsilon, \bar{\nu}(\epsilon)), \epsilon \bar{\nu}(\epsilon)) \\
\lambda & =\lambda_{2} \epsilon^{2}-\epsilon^{4} \bar{\lambda}(\epsilon, \bar{\nu}(\epsilon)) .
\end{aligned}
$$

This ends the proof of the following 


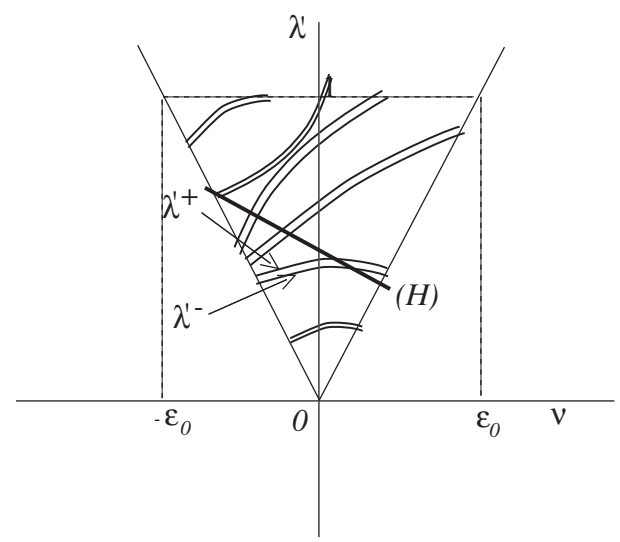

Figure 2: Sketch of the "bad set" in the plane $\left(\nu, \lambda^{\prime}\right)$ for $\epsilon$ fixed. $(H)$ is the "line" given by (68).

Theorem 27. Let $q \geq 4$ be an integer and let $d=2\left(l_{0}+1\right)$ be the dimension of the $\mathbb{Q}$-vector space spanned by the wave vectors $\mathbf{k}_{j}, j=1, \ldots, 2 q$. Moreover, assume that Condition 2 is verified. Then, there exists $s_{0}>d / 2, \epsilon_{0}>0$, and $0<\epsilon_{3}<\epsilon_{0}$ such that, for any $s \geq s_{0}$, $\epsilon<\epsilon_{3}$, there exists a 1-dimensional set $\bar{\Lambda}_{\epsilon}$ of asymptotic full measure as $\epsilon$ tends to 0 with the following property : for any $\epsilon<\epsilon_{3}$, there exist $\bar{\nu}(\epsilon) \in\left[-\epsilon_{0}, \epsilon_{0}\right]$, and $\bar{\lambda}_{\epsilon}:=\bar{\lambda}(\epsilon, \bar{\nu}(\epsilon)) \in \bar{\Lambda}_{\epsilon}$ such that the steady Swift-Hohenberg equation, for $\lambda=\lambda_{2} \epsilon^{2}-\epsilon^{4} \bar{\lambda}_{\epsilon}$ admits a quasipattern solution $U$ in $\mathcal{H}_{s}$, invariant under rotations of angle $\pi / q$ of the form

$$
U=\epsilon u_{0}+\epsilon^{3}\left(u_{1}+\bar{\nu}(\epsilon) u_{0}\right)+\mathcal{O}\left(\epsilon^{5}\right),
$$

where $\lambda_{2}=3(2 q-1)>0, \bar{\lambda}_{\epsilon}=-\lambda_{4}-2 \lambda_{2} \bar{\nu}(\epsilon)+O(\epsilon)$. The quasiperiodic function $u_{0}$ spans the kernel of $(1+\Delta)^{2}$, and coefficients $\lambda_{2}, \lambda_{4}, u_{1}$ occurring in formulae above, are the ones defined in the truncated asymptotic expansion of the solution, computed in section 2.2, see also [IR10].

This is a more detailed formulation of Theorem 1 given in the Introduction.

Remark 28. We know from (17) that

$$
\lambda_{2}=\frac{\left\langle u_{0}^{3}, u_{0}\right\rangle_{0}}{\left\langle u_{0}, u_{0}\right\rangle_{0}}=\left\langle u_{0}^{3}, e^{i \mathbf{k}_{1} \cdot \mathbf{x}}\right\rangle_{0}=3(2 q-1) .
$$

Now, looking at formula (49) for $\alpha_{j}^{(N)}\left(\epsilon, \lambda^{\prime}, \nu\right)$ we might look at the generic case of simple eigenvalues $\mu_{j}^{(N)}\left(\epsilon, \lambda^{\prime}, \nu\right)$. In such a case $\mu_{j}^{(N)}\left(\epsilon, \lambda^{\prime}, \nu\right)$ as well as eigenvectors $\zeta_{j}^{(N)}\left(\epsilon, \lambda^{\prime}, \nu\right)$ are $C^{1}$ function of their arguments (for $N$ fixed). As $\epsilon \rightarrow 0$, the eigenvector tends toward an expression of the form (this form results from the invariance under rotations $R_{s \pi / q}$ ):

$$
\zeta_{j}^{(N)}(0)=\frac{1}{\sqrt{2 q}} \sum_{s=1,2, . .2 q} e^{i R_{s \pi / q} \mathbf{k}_{0} \cdot \mathbf{x}}
$$


which is an eigenvector of the operator $\Pi_{N} \Pi_{0}(1+\Delta)^{2}$ for the eigenvalue $\left(1-\left|\mathbf{k}_{0}\right|^{2}\right)^{2}$ very close to 0 .

Then we have

$$
\begin{aligned}
\left\langle u_{0}^{2} \zeta_{j}^{(N)}(0), \zeta_{j}^{(N)}(0)\right\rangle_{0} & =\frac{1}{2 q}\left\langle u_{0}^{2} \sum_{s=1,2, . .2 q} e^{i R_{s \pi / q} \mathbf{k}_{0} \cdot \mathbf{x}}, \sum_{s=1,2, . .2 q} e^{i R_{s \pi / q} \mathbf{k}_{0} \cdot \mathbf{x}}\right\rangle_{0} \\
& =\left\langle u_{0}^{2} \sum_{s=1,2, . .2 q} e^{i R_{s \pi / q} \mathbf{k}_{0} \cdot \mathbf{x}}, e^{i \mathbf{k}_{0} \cdot \mathbf{x}}\right\rangle_{0} .
\end{aligned}
$$

Now, we notice that

$$
u_{0}^{2}=\sum_{r, l=1,2, \ldots, 2 q} e^{i\left(\mathbf{k}_{r}+\mathbf{k}_{l}\right) \cdot \mathbf{x}},
$$

so that the only terms giving a contribution in the last scalar product are such that

$$
\mathbf{k}_{r}+\mathbf{k}_{l}=\mathbf{k}_{0}-R_{s \pi / q} \mathbf{k}_{0}
$$

for some $r, l, s \in\{1, . ., 2 q\}$. Trivial solutions are $s=2 q, l=r+q$ (corresponds to the constant terms in $u_{0}^{2}$ ) which gives $2 q$ terms equal to 1 . In fact, there are no other solutions: each side of (71) is the sum of two vectors with the same length, so they have the same bisectrix. Since the length of $\mathbf{k}_{0}$ and of $-R_{s \pi / q} \mathbf{k}_{0}$ is close to 1 , and the length of $\mathbf{k}_{r}$ and $\mathbf{k}_{l}$ is 1 , the angle between $\mathbf{k}_{r}$ and $\mathbf{k}_{l}$ and the angle between $\mathbf{k}_{0}$ and of $-R_{s \pi / q} \mathbf{k}_{0}$ are nearly the same. But, this angle should be a multiple of $\pi / q$, so the two pairs of vectors make the same angle between them. Now we see that there is no solution, because of the difference between the lengths of the two pairs (except if the sum is 0). Finally, this shows that

$$
\left\langle u_{0}^{2} \zeta_{j}^{\left(N_{n}\right)}(0), \zeta_{j}^{\left(N_{n}\right)}(0)\right\rangle_{0}=2 q,
$$

This implies that Condition 2 is realized at $\epsilon=0$ for any $q$.

Remark 29. We might be tempted to adapt the parameter $\epsilon$ by defining a new parameter $\epsilon^{\prime}$ as

$$
\epsilon^{\prime}:=\epsilon+\epsilon^{2} \nu(\epsilon) \text {. }
$$

Then it is easy to check (using (68): $\epsilon \bar{\lambda}+2 \nu \lambda_{2}=-\epsilon \lambda_{4}+\mathcal{O}\left(\epsilon^{2}\right)$ ) that the solution $(U, \lambda)$ may be expressed as

$$
\begin{aligned}
U & =\epsilon^{\prime} u_{0}+\epsilon^{\prime 3} u_{1}+\mathcal{O}\left(\epsilon^{\prime 5}\right), \\
\lambda & =\epsilon^{\prime 2} \lambda_{2}+\epsilon^{\prime 4} \lambda_{4}+\mathcal{O}\left(\epsilon^{\prime 5}\right) .
\end{aligned}
$$

This is the form of the power series of the solution which is formally obtained (see subsection 2.2), here after a scaling on $\epsilon$ (which does not change the curve). However we observe that (72) is not invertible, i.e. $\epsilon$ cannot be expressed in term of $\epsilon^{\prime}$ since the choice of $\nu$ is not completely determined in function of $\epsilon$. The mapping $\epsilon \mapsto \epsilon^{\prime}$ is not an homeomorphism. The result is that $\epsilon^{\prime}$ does not take a continuum of values, leading to "holes" in the curve expressed by the series above truncated after the two first terms. These holes are of smaller and smaller size, as $\epsilon \rightarrow 0$. The higher order terms that we do not precisely express, mean that the bifurcating set is centered about the curve above mentioned, and lies in a hornlike region, with the vertex (or the tip) at the bifurcation point $\left(\epsilon^{\prime}=0\right)$. 
Remark 30. The above result in Theorem 1 may be extended in using the formal asymptotic expansion of $U$ truncated at an arbitrary order:

$$
\begin{aligned}
U_{\epsilon} & =\epsilon u_{0}+\epsilon^{3} u_{1}+\ldots+\epsilon^{2 n+1} u_{n}, \\
\lambda_{\epsilon} & =\epsilon^{2} \lambda_{2}+\epsilon^{4} \lambda_{4}+\ldots+\epsilon^{2 n} \lambda_{2 n}
\end{aligned}
$$

and introduce $\left(V, \lambda^{\prime}\right)$ as

$$
\begin{aligned}
U & =U_{\epsilon}+\nu \epsilon^{2 n} u_{0}+3 \nu \epsilon^{2 n+2} u_{1}+\epsilon^{2 n+2} V, \\
\lambda & =\lambda_{\epsilon}-\epsilon^{2 n+1} \lambda^{\prime} .
\end{aligned}
$$

Then we can proceed in the same way as before, and find (exercise left to the courageous reader) $V\left(\epsilon, \lambda^{\prime}, \nu\right)$ and there are solutions $\lambda^{\prime}$ in the good set with

$$
\lambda^{\prime}+2 \nu \lambda_{2}=-\epsilon \lambda_{2 n+2}+\mathcal{O}\left(\epsilon^{2}\right) .
$$

Moreover, if we set

$$
\epsilon^{\prime}=: \epsilon+\epsilon^{2 n} \nu(\varepsilon), \text { (a priori not invertible) }
$$

then we find again the same asymptotic expansion for the bifurcating set:

$$
\begin{aligned}
U & =\epsilon^{\prime} u_{0}+\epsilon^{\prime 3} u_{1}+\ldots+\epsilon^{\prime 2 n+1} u_{n}+\mathcal{O}\left(\epsilon^{\prime 2 n+3}\right) \\
\lambda & =\epsilon^{\prime 2} \lambda_{2}+\epsilon^{\prime 4} \lambda_{4}+\ldots+\epsilon^{\prime 2 n} \lambda_{2 n}+\epsilon^{\prime 2 n+2} \lambda_{2 n+2}+\mathcal{O}\left(\epsilon^{\prime 2 n+3}\right) .
\end{aligned}
$$

\section{A Berti-Bolle-Procesi's version of Nash-Moser theorem}

In this section, we recall notations and the main result of Berti-Bolle-Procesi's paper [BBP10].

Let us consider a scale of Banach spaces $\left(X_{s},\|.\|_{s}\right)$ such that, for all $s \leq s^{\prime}$, we have $X_{s^{\prime}} \subseteq X_{s},\|u\|_{s} \leq\|u\|_{s^{\prime}}$ whenever $u \in X_{s^{\prime}}$. Let us define $X:=\cap_{s \geq 0} X_{s}$. Let $\left(E_{N}\right)_{N \in \mathbb{N}}$ be an increasing family of closed subspaces of $X$ such that $\cup E_{N}$ is dense in $X_{s}$ for every $s \geq 0$. Let us consider projectors $\Pi_{N}: X_{0} \rightarrow E_{N}$ such that, for all $s \geq 0$ and $d \geq 0$ :

- (S1) $\left\|\Pi_{N} u\right\|_{s+d} \leq C(s, d) N^{d}\|u\|_{s}$ if $u \in X_{s}$,

- (S2) $\left\|\left(I-\Pi_{N}\right) u\right\|_{s} \leq C(s, d) N^{-d}\|u\|_{s+d}$ if $u \in X_{s+d}$

Let us consider a $C^{2}$-map $F:\left[0, \epsilon_{0}\left[\times \Lambda \times X_{s_{0}+\nu} \rightarrow X_{s_{0}}\right.\right.$ where $s_{0} \geq 0, \epsilon_{0}>0, \nu>0$ and $\Lambda$ be a bounded domain of $\mathbb{R}^{q}$. We assume, there exists $S \geq s_{0}$ such that for all $s_{0} \leq s<S$, for all $u \in X_{s+\nu}$ with $\|u\|_{s_{0}} \leq 2$ and for all $(\epsilon, \lambda) \in\left[0, \epsilon_{0}[\times \Lambda\right.$ :

- (F1) $F(0, \lambda, 0)=0$, for all $\lambda \in \Lambda$

- (F2) $\max \left(\left\|\partial_{\epsilon} F(\epsilon, \lambda, u)\right\|_{s},\left\|\partial_{\lambda} F(\epsilon, \lambda, u)\right\|_{s}\right) \leq C(s)\left(1+\|u\|_{s+\nu}\right)$ and $\left\|D_{u} F(\epsilon, \lambda, 0) h\right\|_{s} \leq$ $C(s)\|h\|_{s+\nu}$

- (F3) $\left\|D_{u}^{2} F(\epsilon, \lambda, u)(h, v)\right\|_{s} \leq C\left(\|u\|_{s+\nu}\|h\|_{s_{0}}\|v\|_{s_{0}}+\|v\|_{s+\nu}\|h\|_{s_{0}}+\|v\|_{s_{0}}\|h\|_{s+\nu}\right)$ 
- (F4) $\max \left(\left\|\partial_{\lambda} D_{u} F(\epsilon, \lambda, u) h\right\|_{s},\left\|\partial_{\epsilon} D_{u} F(\epsilon, \lambda, u) h\right\|_{s}\right) \leq C(s)\left(\|u\|_{s+\nu}\|h\|_{s_{0}}+\|h\|_{s+\nu}\right)$

Let us define $L_{N}(\epsilon, \lambda, u):=\Pi_{N} D_{u} F(\epsilon, \lambda, u)_{\mid E_{n}}$. Let us consider two nonnegative parameters $\mu, \sigma$ such that

$$
\sigma>4(\mu+\nu), \quad \bar{s}:=s_{0}+4(\mu+\nu+1)+2 \sigma<S
$$

For all $\gamma>0$, we define

$$
\begin{aligned}
J_{N, \gamma, \mu} & \subseteq\left\{( \epsilon , \lambda , u ) \in \left[0, \epsilon_{0}\left[\times \Lambda \times E_{N} \mid \forall s \in\left[s_{0}, \bar{s}\right], \forall h \in E_{N},\right.\right.\right. \\
& \left.\left\|L_{N}^{-1}(\epsilon, \lambda, u) h\right\|_{s} \leq \frac{N^{\mu}}{\gamma}\left(\|h\|_{s}+\|u\|_{s}\|h\|_{s_{0}}\right)\right\},
\end{aligned}
$$

where, in the above definition, $L_{N}(\epsilon, \lambda, u)$ is implicitly supposed to be invertible. Given $K>0$, we define

$$
\mathcal{U}_{N, K}:=\left\{u \in C ^ { 1 } \left(\left[0, \epsilon_{0}\left[\times \Lambda, E_{N}\right) \mid u(0, \lambda)=0,\|u\|_{s_{0}} \leq 1, \max \left(\left\|\partial_{\lambda} u\right\|_{s_{0}},\left\|\partial_{\epsilon} u\right\|_{s_{0}}\right) \leq K\right\} .\right.\right.
$$

Remark 31. In Berti-Bolle's paper, the condition $u(0, \lambda)=0$ is not written explicitly in the definition of $\mathcal{U}_{N, K}$ but it is used.

For all $u \in \mathcal{U}_{N, K}$, we set

$$
G_{N, \gamma, \mu}(u):=\left\{( \epsilon , \lambda ) \in \left[0, \epsilon_{0}\left[\times \Lambda\left[\mid(\epsilon, \lambda, u(\epsilon, \lambda)) \in J_{N, \gamma, \mu}\right\} .\right.\right.\right.
$$

We assume that there exists $\mu \geq 0$, and $\sigma \geq 0$ satisfying (73), there exists $\bar{\gamma}>0, M \in \mathbb{N}^{*}$ and $C>0$ such that

1.

$$
\text { (L1) } \left.\left.\left.\forall(\epsilon, \gamma) \in] 0, \epsilon_{0}\right] \times\right] 0, \bar{\gamma}\right], \quad \operatorname{meas}_{\mathbb{R}^{q+1}}\left(G_{N, \gamma, \mu}(0)^{c} \cap([0, \epsilon[\times \Lambda)) \leq C \gamma \epsilon .\right.
$$

2. (L2) For all $\gamma \in] 0, \bar{\gamma}]$ and for all $\bar{K}>0$, there exists a positive $\tilde{\epsilon}(\gamma, \bar{K})<\epsilon_{0}$ such that, if $0<\epsilon<\tilde{\epsilon}$, if the integers $N_{1}, N_{2}$ satisfies $M \leq N_{1} \leq N_{2}$, and if $u_{i} \in \mathcal{U}_{N_{i}, \bar{K}}$, $i=1,2$ satisfy $\left\|u_{1}-u_{2}\right\|_{s_{0}} \leq N_{1}^{-\sigma}$ then

$$
\operatorname{meas}_{\mathbb{R}^{q+1}}\left(( G _ { N _ { 2 } , \gamma , \mu } ( u _ { 2 } ) ) ^ { c } \backslash ( G _ { N _ { 1 } , \gamma , \mu } ( u _ { 1 } ) ) ^ { c } \cap \left([0, \epsilon[\times \Lambda)) \leq C \frac{\gamma \epsilon}{N_{1}} .\right.\right.
$$

Let us recall the main theorem of Berti-Bolle-Procesi's paper :

Theorem 32. [BBP10][theorem 1] Assume that condition $(F 1),(F 2),(F 3),(F 4),(L 1),(L 2)$ and (73) are satisfied. Then there exists $C>0$ and for all $0<\gamma<\bar{\gamma}$, there exists $0<\epsilon_{3}(\gamma)<\epsilon_{0}$ and $a C^{1}$-map

$$
u:\left[0, \epsilon_{3}\left[\times \Lambda \rightarrow X_{s_{0}+\nu}\right.\right.
$$

such that for all $(\epsilon, \lambda) \in\left[0, \epsilon_{3}\left[\times \Lambda \backslash C_{\gamma}, u(0, \lambda)=0\right.\right.$ and $F(\epsilon, \lambda, u(\epsilon, \lambda))=0$ where the Lebesgue measure of the set $C_{\gamma}$ satisfies meas $\mathbb{R}^{q+1}\left(\left[0, \epsilon\left[\times \Lambda \cap C_{\gamma}\right) \leq C \gamma \epsilon\right.\right.$ if $\epsilon \leq \epsilon_{3}$.

Acknowledgments: The authors warmly thank Massimiliano Berti and Michela Procesi for the interactions they had about this work, specially during the Winter school at St Etienne de Tinée in February 2015. The authors thank also B.W.Rink and both very pertinent referees, for pointing out mistakes in previous versions. 


\section{References}

[AB15] T.Alazard, P.Baldi. Gravity capillary standing water waves. Arch. Rat. Mech. Anal. (2015). DOI:10.1007/s00205-015-0842-5.

[AG12] M. Argentina and G.Iooss. Quasipatterns in a parametrically forced horizontal fluid film. PhysicaD: Nonlinear Phenomena, 241, 16:1306-1321, 2012.

[BB10] M.Berti, P.Bolle. Sobolev periodic solutions of nonlinear wave equations in higher spatial dimensions. Arch. Ration. Mech. Anal. 195, 2 (2010) 609-642.

[BBP10] M. Berti, P. Bolle, and M. Procesi. An abstract Nash-Moser theorem with parameters and applications to PDEs. Ann. Inst. H. Poincaré Anal. Non Linéaire, 27(1):377-399, 2010.

[BCP] M.Berti, L.Corsi, M.Procesi. An abstract Nash-Moser theorem and quasi-periodic solutions for NLW and NLS on compact Lie groups and homogeneous manifolds.Com. Math. Phys.2014. DOI 10.1007/s00220-014-2128-4

[Bou95] J. Bourgain. Construction of periodic solutions of nonlinear wave equations in higher dimension. Geom. Funct. Anal., 5(4):629-639, 1995.

[BIS] B.Braaksma, G.Iooss, L.Stolovitch. Existence of quasipatterns solutions of the Swift-Hohenberg equation. Arch. Rat. Mech. Anal. 209, 1 (2013), 255-285. Erratum ARMA 211, 3 (2014), 1065.

[Cra00] W.Craig. Problèmes de petits diviseurs dans les équations aux dérivées partielles, volume 9 of Panoramas et Synthèses [Panoramas and Syntheses]. Société Mathématique de France, Paris, 2000.

[BCM92] P. Alstrom B. Christiansen and M.T.Levinsen. Ordered capillary-wave states: Quasi-cristals, hexagons,and radial waves. Phys. rev. Lett., 68:2157-2160, 1992.

[EF94] W. S. Edwards and S. Fauve. Patterns and quasi-patterns in the Faraday experiment. J. Fluid Mech., 278:123-148, 1994.

[IPT05] G.Iooss, P.Plotnikov, J.F.Toland. Standing waves on an infinitely deep perfect fluid under gravity. Arch. Rat. Mech. Anal. 177, 367-478 (2005).

[IP09] G.Iooss, P.Plotnikov. Small divisor problem in the theory of three-dimensional water gravity waves. Mem. Am. Math. Soc. 200, 940 (2009).

[IP11] G.Iooss, P.Plotnikov. Asymmetrical three-dimensional travelling gravity waves. Arch. Rat. Mech. Anal. 200 (2011) 789-880.

[IR10] G. Iooss and A. M. Rucklidge. On the existence of quasipattern solutions of the Swift-Hohenberg equation. J. Nonlinear Sci., 20(3):361-394, 2010.

[Kato] Tosio Kato. Perturbation theory for linear operators. Classics in Mathematics. Springer-Verlag, Berlin, 1995. Reprint of the 1980 edition. 
[Wash97] L.C.Washington. Introduction to cyclotomic fields. Vol 83, Graduate Texts in Mathematics. Springer-Verlag. New york, 2nd ed. 1997. 